\title{
Horizontal gene transfer contributes to virulence and antibiotic resistance of Vibrio harveyi 345 based on complete genome sequence analysis
}

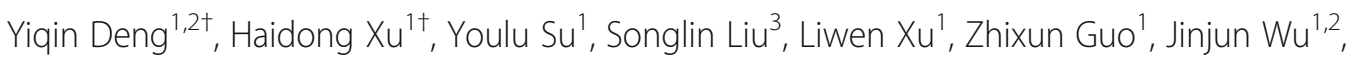
Changhong Cheng ${ }^{1}$ and Juan Feng ${ }^{1,2^{*}}$ (D)

\begin{abstract}
Background: Horizontal gene transfer (HGT), which is affected by environmental pollution and climate change, promotes genetic communication, changing bacterial pathogenicity and drug resistance. However, few studies have been conducted on the effect of HGT on the high pathogenicity and drug resistance of the opportunistic pathogen Vibrio harveyi.

Results: V. harveyi 345 that was multidrug resistant and infected Epinephelus oanceolutus was isolated from a diseased organism in Shenzhen, Southern China, an important and contaminated aquaculture area. Analysis of the entire genome sequence predicted 5678 genes including 487 virulence genes contributing to bacterial pathogenesis and 25 antibiotic-resistance genes (ARGs) contributing to antimicrobial resistance. Five ARGs (tetm, tetb, anrs, dfra17, and sul2) and one virulence gene (CU052_28670) on the pAQU-type plasmid p345-185, provided direct evidence for HGT. Comparative genome analysis of $31 \mathrm{~V}$. harveyi strains indicated that 217 genes and 7 gene families, including a class $C$ beta-lactamase gene, a virulence-associated protein $\mathrm{D}$ gene, and an OmpA family protein gene were specific to strain $\mathrm{V}$. harveyi 345. These genes could contribute to HGT or be horizontally transferred from other bacteria to enhance the virulence or antibiotic resistance of 345. Mobile genetic elements in 71 genomic islands encoding virulence factors for three type III secretion proteins and 13 type VI secretion system proteins, and two incomplete prophage sequences were detected that could be HGT transfer tools. Evaluation of the complete genome of $V$. harveyi 345 and comparative genomics indicated genomic exchange, especially exchange of pathogenic genes and drug-resistance genes by HGT contributing to pathogenicity and drug resistance. Climate change and continued environmental deterioration are expected to accelerate the HGT of $V$. harveyi, increasing its pathogenicity and drug resistance.
\end{abstract}

Conclusion: This study provides timely information for further analysis of $V$. harveyi pathogenesis and antimicrobial resistance and developing pollution control measurements for coastal areas.

Keywords: Vibrio harveyi, Virulence, Antibiotic resistance, Comparative genomics, Horizontal gene transfer

\footnotetext{
* Correspondence: juanfeng@scsfri.ac.cn

Yiqin Deng and Haidong Xu Joint first author

${ }^{1}$ Key Laboratory of South China Sea Fishery Resources Exploitation \&

Utilization, Ministry of Agriculture and Rural Affairs, South China Sea Fisheries

Research Institute, Chinese Academy of Fishery Sciences, Guangzhou 510300,

China

${ }^{2}$ Tropical Aquaculture Research and Development Centre, South China Sea

Fisheries Research Institute, Chinese Academy of Fishery Sciences, Hainan

572426, China

Full list of author information is available at the end of the article
}

(c) The Author(s). 2019 Open Access This article is distributed under the terms of the Creative Commons Attribution 4.0 International License (http://creativecommons.org/licenses/by/4.0/), which permits unrestricted use, distribution, and reproduction in any medium, provided you give appropriate credit to the original author(s) and the source, provide a link to the Creative Commons license, and indicate if changes were made. The Creative Commons Public Domain Dedication waiver (http://creativecommons.org/publicdomain/zero/1.0/) applies to the data made available in this article, unless otherwise stated. 


\section{Background}

With the rapid expansion of aquaculture, vibriosis has become one of the most serious diseases to endanger sustainable aquaculture development $[1,2]$. Vibrio harveyi is a halophilic, luminescent Gram-negative $\gamma$-proteobacteria, as an important pathogen of vibriosis [3-5]. V. harveyi has been extensively studied for more than a decade and is reported to be a serious pathogen for a range of marine vertebrates and invertebrates [4-7]. The earliest report of $V$. harveyi causing serious infections was to common snook (Centropomus undecimalis) in Florida, USA [8]. Subsequently, $V$. harveyi was shown to cause disease in Penaeus monodon and Penaeus japonicas in Thailand [10], brownspotted grouper in Kuwait [9], common dentex (Dentex dentex) in Spain [10], Holothuria scabra (Holothuroidea, Echinodermata) in Toliara, Madagascar [11], Asian seabass (Lates calcarifer) in Malaysia [12], and Epinephelus spp. in China [13-15].

The wide use of antibiotics, increased environmental pollution and global climate change are leading to the enhancement of drug resistance of Vibrio spp., including $V$. harveyi. Several studies show that drug resistance and multidrug resistance (MDR) are occurring in aquaculture worldwide, including in shrimp ponds in Thailand, Malaysia, India, and China [16-19]; shellfish farms in Malaysia, Korea, USA, Poland and China [20-24]; and fish farms in Italy, Korea, and China [25-27]. Enhanced drug resistance leads to stronger virulence resulting in difficulty in preventing and treating $V$. harveyi infection $[15,16]$. For example, Nakayama et al. [28] found that gradually increasing antibiotic concentration and frequent subculturing enhances $V$. harveyi antibiotic resistance, elevating the toxicity of $V$. harveyi. The pathogenic and drug-resistant genes of $V$. harveyi are a key to the fundamental cause of pathogenicity and drug resistance. Therefore, studying the pathogenic and drug-resistance genes of $V$. harveyi will provide an important foundation for determining pathogenic and drug-resistance mechanisms.

Although $V$. harveyi a recognized pathogen of marine animals, different strains vary in their ability to cause disease [29]. The main virulence factors of $V$. harveyi are extracellular proteases, outer membrane proteins, hemolysins, esterases, phospholipases, exotoxin and secretion systems $[29,30]$. Production of antimicrobial enzymes that inactivate antibacterial drugs is an important resistant mechanism [31]. Inactivating enzymes can be expressed by genes on plasmids or chromosomes [32] Virulence factors and antibiotic-resistance genes (ARGs) can also be vertically transferred and spread via horizontal gene transfer (HGT) through mobile genetic elements (MGEs) such as plasmids, bacteriophages, transposons, integrative and conjugative elements (ICEs), and genetic islands (GIs) [33]. Park et al. [34] found that MGEs that are prophages, GIs and pathogenicity islands carry different combinations of virulence factors that promote immune evasion and superantigens that contribute to serious Staphylococcus aureus infection. Le et al. [35] reported that $0.92 \%$ (36 of 38,895) analyzed proteins from 31 Rickettsiales genomes are associated with strong bootstrap support for HGT with function as ATPases, aldolases, transporter activities, cystathionine beta-lyases, sugar phosphate permeases, growth inhibitors and antitoxin activities. For horizontal transfer, exogenous DNA must evade the bacterial immune system such as restriction modification systems (RM systems) and CRISPR-Cas systems [36, 37].

Serious infection and multidrug resistance lead to the emergency of the availability of whole genome sequences for different $V$. harveyi strains to determine pathogenic and antimicrobial-resistance mechanisms. Evidence is growing that HGT is an important driving force for prokaryotic evolution affecting pathogenicity and drug resistance [34, 38]. Coastal urbanization has recently intensified, resulting in the production of a large amount of antibiotics, heavy metals, and nutrients pollutants (Additional file 1: Table S1), which may increase the pathogenicity and drug resistance of $V$. harveyi by affecting HGT [39, 40]. $V$. harveyi is the dominant species that causes serious infection and mortality of farmed fish in Guangdong, Southern China [14, 15]. However, little information is available on the mechanism of $V$. harveyi pathogenesis and drug resistance.

The predominant strain $V$. harveyi 345 is multidrug resistant to ampicillin, rifampicin, tetracycline, pediatric compound sulfamethoxazole tablets, vancomycin, doxycycline, trimethoprim, streptomycin, kanamycin, sulfamethoxazole, furazolidone, cefixime, and chloramphenicol. This strain was isolated from a diseased E. oanceolutus in Shenzhen, Southern China. V. harveyi 345 is suspected to lead to kidney enlargement and softening, spleen enlargement, and anal bleeding of $E$. oanceolutus and has a median lethal dose $\left(\mathrm{LD}_{50}\right)$ of $9.83 \times 10^{5} \mathrm{CFU} \cdot \mathrm{g}^{-1}$ [41]. Therefore, its complete genome information and HGT events are helpful for clarifying $V$. harveyi pathogenesis and drug resistance, controlling $V$. harveyi disease and reducing economic losses. In this study, we present the entire genome sequence of $V$. harveyi 345 with comparative genomics analysis of its pathogenesis, antimicrobial resistance and genome expansion caused by HGT.

\section{Results}

\section{General features of $V$. harveyi 345}

A total of 1189 megabases clean data and 98,602 subreads were generated assembling into two contigs with average genome coverage of $41.71 \times$. After error correction, GATK analysis and gap filling, a genome of 6,185, 822 bp was generated, which was smaller than comparable strains KC13.17.5, E385, and 74F and larger than 27 
other comparable strains (Additional file 2: Table S2). The chromosomal G $+C$ content (44.76\%) of 345 agreed with values from 30 comparable strains (44.60-45.60\%) (Additional file 2: Table S2). Combined sequencing analysis revealed that the complete genome of $V$. harveyi 345 contained two circular chromosomes, named ChI ( 3 , 713,225 bp, 44.81\% G + C, CP025537) and ChII (2,220, $396 \mathrm{bp}, 44.81 \% \mathrm{G}+\mathrm{C}$, CP025538), and two circular megaplasmids named p345-185 (185,327 bp, 43.48\% $\mathrm{G}+\mathrm{C}, \mathrm{CP} 025539)$ and p345-67 (66,874 bp, 43.48\% G + C, CP025540) (Table 1 and Fig. 1).

\section{Gene annotation}

A total of 5678 genes (fewer than ZJ0603 and more than 29 other strains) (Additional file 2: Table S2) were predicted to be an average 929 bp with a mean GC content of $45.68 \%$, accounting for $85.29 \%$ of the genome. The three highest levels of gene length distribution were $400-500 \mathrm{bp}, 600-700 \mathrm{bp}$, and $300-400 \mathrm{bp}$ and the three lowest were $0-100 \mathrm{bp}, 1900-2000 \mathrm{bp}$, and $1800-1900 \mathrm{bp}$ (Fig. 2a). In addition, 128 tRNA, 31 rRNA and 13 sRNA genes were identified with confidence. A total of 89 tandem repeats, including 44 minisatellite DNA and 35 microsatellite DNA, were predicted (Table 1 and Fig. 1).

Predicted open reading frames (ORFs) were further classified into Clusters of Orthologous Groups (COG) (4586 ORFs in total contenting 80.77\%, Fig. 2b). According to COG categorization analysis, the top five groups in abundance were amino acid transport and metabolism (361 ORFs), transcription (350 ORFs), general function prediction only (343 ORFs), signal transduction mechanisms (312 ORFs) and carbohydrate transport and metabolism (312 ORFs) (Fig. 2b). In addition, 3247 genes were classified into 40 functional Kyoto Encyclopedia of Genes and Genomes (KEGG) pathways (Additional file 3: Figure S1). Some genes were involved in more than one KEGG pathway and included the top five pathways for replication and repair (784 ORFs), carbohydrate metabolism (633 ORFs), signal transduction (506 ORFs), infectious diseases (463 ORFs) and membrane transport (432 ORFs). Furthermore, 3453 genes were classified into 40 functional Gene Ontology (GO) classifications (Additional file 4: Figure S2). Some genes were involved in more than one GO classifications and included the top five classifications of metabolic process (1914 ORFs), cellular process (1881 ORFs), catalytic activity (1785 ORFs), single-organism process (1526 ORFs) and binding (1494 ORFs).

Twelve RM system genes were annotated in $V$. harveyi strain 345 (Table 2). Ten belonged to Type I RM systems, encoding three $\mathrm{R}$ subunits ( $h s d R$ ), two $\mathrm{S}$ subunits (hsdS) and five $\mathrm{M}$ subunits $(h s d M)$. The other two belonged to type III RM systems, encoding a methyltransferase $(\mathrm{mod})$ and a restriction enzyme (res).

\section{Virulence factors}

A total of 487 putative virulence factors were predicted (Table 3 and Additional file 5: Table S3), involving adherence (type IV pili, lipooligosaccharide LOS, and OmpU), motility (polar flagellar proteins, chemotaxis proteins, lateral flagellin proteins, and motor proteins), regulation (Autoinducer-2 and cholerae autoinducer-1), secretion system (type II/III/IV/VI secretion system proteins, T2/3/4/6SS proteins), and iron uptake. Many genes were identical to genera other than Vibrio such as Escherichia, Pseudomonas, Salmonella, Aeromonas, and Mycobacterium. T3SS1, which is widely reported to be involved in Vibrio pathogenesis, was found on ChI. T6SSs are implicated in cell targeting and virulence, and are believed to mediate antibacteria [42]. Six T6SS proteins on Hcp secretion island-1 were detected. The th gene encoding a thermolabile hemolysin (TLH) was on ChII. In addition, four virulence genes (CU052_28670, CU052 00025, CU052_00190, and CU052_00400) were on plasmid with CU052_28670 on p345-185, and CU052_00025, CU052_00190, and CU052_00400 on p345-67.

\section{Antimicrobial-resistance genes}

We identified 25 genes predicted to have $>40 \%$ identity to well-characterized ARGs (Table 4), including genes

Table 1 Genome features of $V$. harveyi 345

\begin{tabular}{lllll}
\hline Features & Chl & Chll & Plasmid p345-185 & Plasmid p345-67 \\
\hline Length (bp) & $3,713,225$ & $2,220,396$ & 185,327 & 66,874 \\
G + C content (\%) & 44.81 & 44.81 & 43.48 & 43.48 \\
Genes & 3345 & 2044 & 208 & 81 \\
rRNA gene & 28 & 3 & 0 & 0 \\
tRNA genes & 113 & 15 & 0 & 0 \\
sRNA genes & 6 & 7 & 0 & 0 \\
Minisatellite DNA & 26 & 15 & 2 & 1 \\
Microsatellite DNA & 22 & 11 & 1 & 1 \\
GenBank accession no. & CP025537 & CP025538 & CP025539 \\
\hline
\end{tabular}



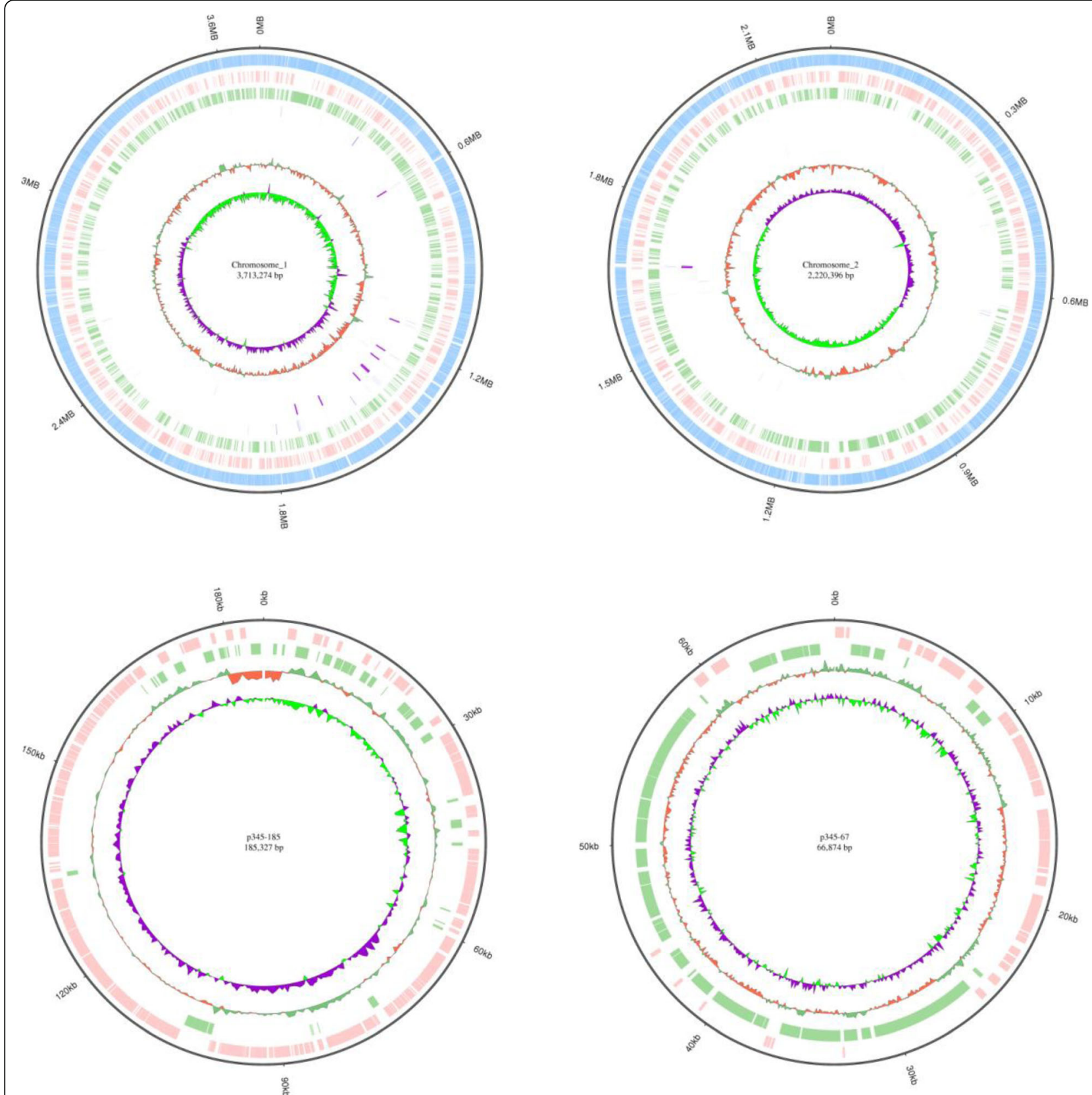

Fig. 1 Circular representation of the $V$. harveyi 345 genome. Inner to outer: genome size, all annotated genes, forward-strand genes, reversestrand genes, tRNA, rRNA, sRNA, GC and GC-skew

for aminoglycoside (golc, adeb, emre), penicillin ( $p b p 2$, pbp1a, pbp1b, pbp2), tetracycline (tet34, tetm, tetb), chloramphenicol (adeb, ceob, $m d t l$ ) and trimethoprim resistance (dfra26). In addition, five genes (CU052 28095, CU052_28120, CU052_28525, CU052_28540, and CU052_29140) were on the plasmid p345-185. These results were consistent with antibiotic-susceptibility assay data for strain 345 showing resistance to streptomycin, ampicillin, tetracycline, chloramphenicol, trimethoprim, and sulfamethoxazole.

\section{Genomic islands, prophages and CRISPR-Cas systems}

Forty-seven GIs were detected on ChI (Additional file 6: Table S4) and 24 on ChII in $V$. harveyi 345 (Additional file 7: Table S5). In the GIs of ChI, 40 transposases were encoded belonging to the IS110, IS21, IS256, IS3, IS5/IS1182, IS66, IS91, ISL3, and ISNCY families and seven belonging to two GIs. Another eight genes were identified that encoded IS66 family insertion sequence hypothetical proteins. Three integrases and one serine recombinase were encoded. Three genes were 


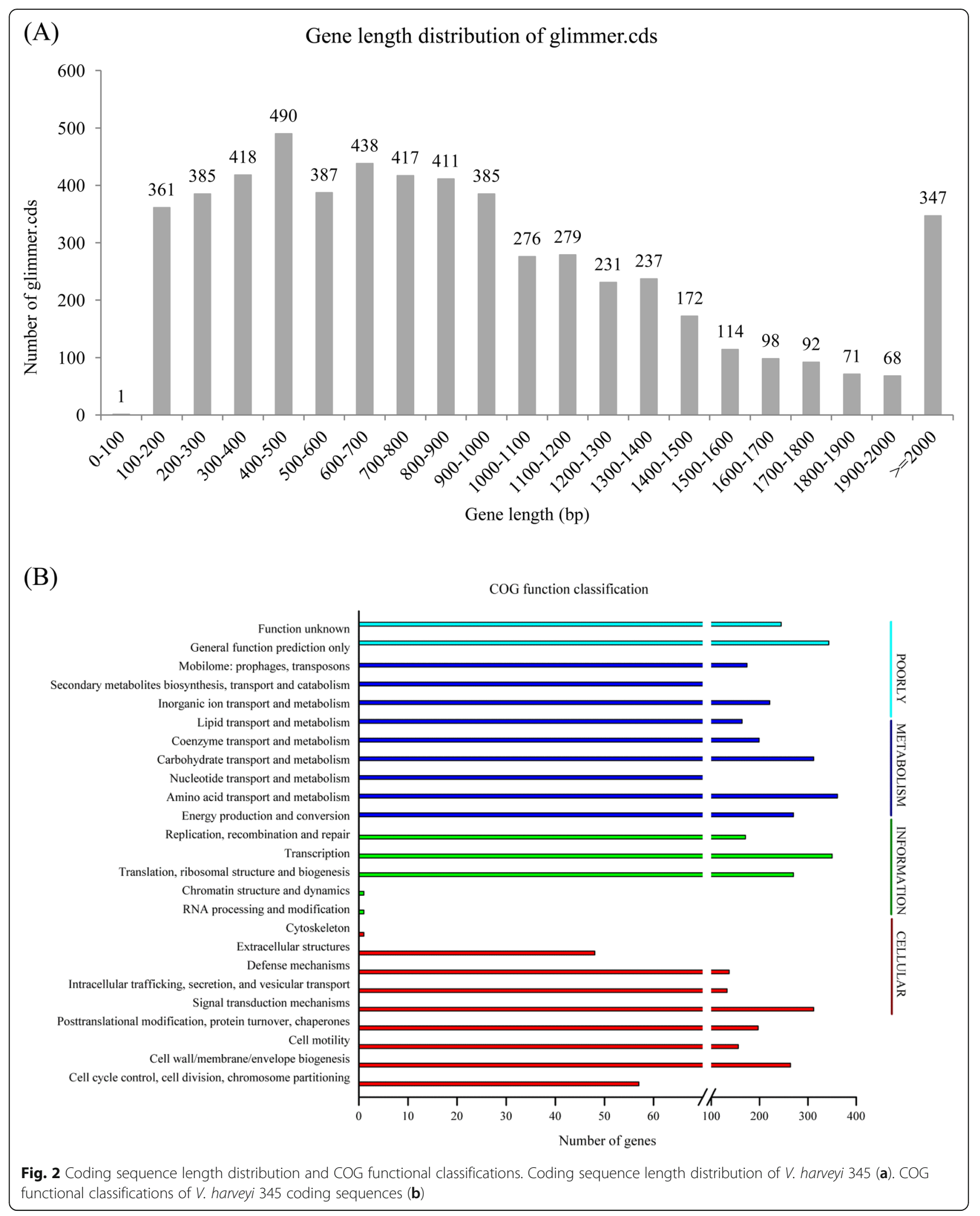


Table 2 The RM system of $V$. harveyi 345

\begin{tabular}{lll}
\hline Gene_id & Gene name & Products \\
\hline CU052_00675 & hsdR & type I restriction enzyme, R subunit \\
CU052_10560 & hsdR & type I restriction enzyme, R subunit \\
CU052_10570 & hsdS & type I restriction enzyme, S subunit \\
CU052_10575 & hsdM & type I restriction enzyme, M protein \\
CU052_17245 & hsdM & type I restriction enzyme, M protein \\
CU052_17265 & hsdR & type I restriction enzyme, R subunit \\
CU052_17270 & hsdS & type I restriction enzyme, S subunit \\
CU052_17275 & hsdM & type I restriction enzyme, M protein \\
CU052_17285 & hsdM & type I restriction enzyme, M protein \\
CU052_27190 & hsdM & type I restriction enzyme, M protein \\
CU052_16835 & mod & adenine-specific DNA-methyltransferase \\
CU052_16840 & res & type III restriction enzyme \\
\hline
\end{tabular}

predicted to encode type III secretion proteins and one was predicted to encode another virulence factor (CU052_RS12915). In the GIs of ChII, nine genes encoding transposases belonging to the IS3 and ISNCY families were detected with four belonging to more than one GI. We found three genes encoding integrases and 13 genes on three GIs predicted to encode type VI secretion system (T6SS) proteins.

Two intact prophage sequences were identified (Additional file 8: Table S6 and no CRISPR elements were predicted.

\section{Phylogenetic analysis, pan-core genes, dispensable and strain-specific genes}

Phylogenetic trees showed that $V$. harveyi 345 closely related to other $V$. harveyi strains, especially $V$. harveyi VHJR7 and V. harveyi CAIM463 (Fig. 3).

The pan-gene refers to the total number of genes in all 31 strains, while core-gene represents orthologous genes shared among them. Dispensable genes were not included in at least one of the 31 strains and strainspecific genes were included in only one strain. Using 31 genomes (Additional file 2: Table S2), 9878 pan-genes with a total size of 2,764,797 amino acid (aa) and 3338 core-genes with a total size of 1,133,465 aa were identified (Fig. 4a-b-c). A dispensable gene heatmap showed that each strain contained strain-specific genes (Fig. 4d). A total of 217 genes were specific to $V$. harveyi 345 with 10 on p345-67, 94 on ChI, 17 on ChII, and 96 on p345185 (Additional file 9: Table S7, Fig. 5a). Twenty-nine genes were annotated by Non-Redundant (NR) database and the others were hypothetical protein-encoding genes (Additional file 9: Table S7, Fig. 5b). Four annotated genes encoded MGEs, including two type IV conjugative transfer proteins (CU052_28715 and CU052_28750) and two recombinases (CU052_29120 and CU052_00050) on p345-185 or p345-67. Four (CU052_13455, CU052 13535, CU052_13525, and CU052_13445) were phagerelated genes on ChI. Four genes (CU052_14535, CU052_14240, CU052_23640, and CU052_13020) on Chr I encoded membrane proteins. One beta-lactamase class C-encoding gene (CU052_00920) was on Chr I. Two DNA methylase-encoding genes (CU052_28385 and CU052_28885) belonging to the bacterial immune system were on p345-185. One virulence-associated protein D (CU052_13320) was on Chr I (Additional file 9: Table S7).

\section{Strain-specific gene family}

A strain-specific gene family was present in one of the 31 strains. Seven gene families were strain specific to $V$. harveyi 345 with $>50 \%$ identity to Pseudovibrio spp., Photobacterium spp., Escherichia spp., and other Vibrio spp. (V. parahaemolyticus) and were involved in recombination, peptide synthesis, conjugation and integration (Table 5).

\section{HGT candidates on chromosomes}

CU052_00920 in $V$. harveyi 345 encoded a betalactamase class $\mathrm{C}$ consisting of 486 amino acids. It shared no similarity with any protein of $V$. harveyi, Vibrio spp. or even $\gamma$-proteobacteria presently known. Instead, it shared more than $40 \%$ identity with the protein in four Pseudovibrio spp. (58.76-59.21\% identity) suggesting recent acquisition of this gene from a Pseudovibrio strain (Fig. 6a and d). Two proteins were annotated, one as a serine hydrolase and the other as a class A beta-lactamase-related serine hydrolase.

CU052_13320 encoded a virulence-associated protein D of 94 amino acids. It shared $40-75.53 \%$ identity with Pseudomonas spp., Aeromonas spp., Neisseria spp., Haemophilus spp., Rodentibacter spp., Alysiella spp., Kingella spp., Haemophilus spp., and Halomonas spp. No similarity with any $V$. harveyi or known Vibrio spp. proteins was detected (data not shown). The top 15 similar strains were selected for muti-alignment and phylogenetic analysis (Fig. 6b and e). CU052_13320 was closest to a virulence factor in Pseudomonas aeruginosa (73.40\% identity) and Aeromonas sobria (75.53\% identity), suggesting HGT of this gene from $P$. aeruginosa or A. sobria (Fig. 6b and e).

The CU052_14535 gene, encoding a 167 amino acid membrane protein in $V$. harveyi 345, was identified as an OmpA family protein in many other homologs. CU052_14535 showed more than 40\% identity with many strains of Photobacterium spp., Salinivibrio spp., Aeromonas spp., Ferrimonas spp., Grimontia spp., Aliivibrio spp., and other Vibrio species, but not with $V$. harveyi (data not shown). The top 15 similar strains were selected for muti-alignment and phylogenetic analysis 
Table 3 Virulence factors predicted with VFDB database

\begin{tabular}{|c|c|c|c|}
\hline Virulence factor & Annotation & Chromosome & Identity genus \\
\hline \multicolumn{4}{|l|}{ Adherence } \\
\hline acfA & ACF & $\|$ & Vibrio \\
\hline$\| p A$ & $\| \mathrm{pA}$ & । & Vibrio \\
\hline VP1611 & MAM7 & I & Vibrio \\
\hline mshH, mshl, mshl, mshJ, mshK, mshL, mshM, mshN, mshE, & MSHA type IV pilus & ।, II & Vibrio \\
\hline \multicolumn{4}{|l|}{$m s h G, m s h F, m s h A, m s h D, m s h A, m s h A, m s h A, m s h A$, } \\
\hline ompU & OmpU & । & Vibrio \\
\hline aatC, aatC & TCP & $\|$ & Escherichia \\
\hline flp-1, flp-1, tadB, tadC, tadA, flp, rcpC, rcpA, tadZ, tadA, & Flp type IV pili & I, II & Vibrio, Aggregatibacter \\
\hline \multicolumn{4}{|l|}{$\operatorname{tad} B, \operatorname{tad} C, \operatorname{tad} D, \operatorname{tad} E, \operatorname{tad} F, \operatorname{tad} G, \operatorname{rcp} A, \operatorname{tad} A$} \\
\hline vfr, rpoN, rpoS, pilW, fimT, pilE, pilM, pilN, pilO, pilP, & type IV pili & I, II & Vibrio, Pseudomonas, Salmonella, \\
\hline pilQ, pilT, pill, pilf, pilT, pilR, pilR, pilR, pill, pilA, & & & Dichelobacter, Yersinia, Haemophilus \\
\hline \multicolumn{4}{|l|}{ pilR, pilR, pil, $R$, pill, pilC, pilB } \\
\hline CJJ81176_1161, CFF8240_1412, CFF8240_1412, & LOS & I, II & Campylobacter, Haemophilus \\
\hline \multicolumn{4}{|l|}{ C8」_1080, kdsB, IpxK, msbA, kdsA, IpxC, gmhA/lpcA, } \\
\hline \multicolumn{4}{|l|}{ htrB, lgtF, lgtF,ops $X / r f a C, k d k A, m s b B$, rfaD, orfM, htrB, } \\
\hline \multicolumn{4}{|l|}{ galE, IpxA, IpxB,gmhAl|pcA, htrB, IpxH, yhxB/manB, } \\
\hline \multicolumn{4}{|l|}{ gale, msbA, rfaE, neuC1, IpxD } \\
\hline tuf, tuf & EF-Tu & I & Mycoplasma \\
\hline$h t p B$ & Hsp60 & । & Legionella \\
\hline lap & $\begin{array}{l}\text { Listeria adhesion } \\
\text { protein }\end{array}$ & । & Listeria \\
\hline СT396, СT396 & MOMP & । & Chlamydia \\
\hline \multicolumn{4}{|l|}{ Motility } \\
\hline flaD, flaA, flaL, flak, flas, flal, flaH, flaG, flaF, flaE, & Flagella & I, II & Vibrio, Pseudomonas, Salmonella, \\
\hline flad, flac, flaB, cheR, cheA, cheB, cheV, cheV, cheW, & & & Legionella, Aeromonas, Pseudomonas \\
\hline \multicolumn{4}{|l|}{ cheW, cheY, cheZ, filM,flaB, flaD, flaD, flaE, flaG, flal, } \\
\hline \multicolumn{4}{|l|}{$f l e Q, f l e Q, f l e R, f l g A, f l g A, f \lg B, f l g C, f \lg D, f \lg E$} \\
\hline \multicolumn{4}{|l|}{$f l g F, f l g G, f l g H, f l g l, f l g J, f l g K, f l g L, f l g M, f l g M$ flgN, } \\
\hline \multicolumn{4}{|l|}{$f l g N$, flgO, flgP, flgT, flhA, flhA, flhB, flhB, flhF, flhG, } \\
\hline \multicolumn{4}{|l|}{ fliA, flid, fliE, fliE, fliF, fliF, fliG, fliG, fliH, fliH, flil, } \\
\hline \multicolumn{4}{|l|}{ flil, flis, flis, flik, fliL, flim, fliN, fliN, fliO, flip, flip, } \\
\hline \multicolumn{4}{|l|}{ fliQ, fliQ, fliR, fliR, flis, fliY, flmH, flmH, flmH, flrA, } \\
\hline \multicolumn{4}{|l|}{ flrB, flrC, lafA, lafB, lafC, lafD, lafE, laff, lafk, } \\
\hline \multicolumn{4}{|l|}{ lafs, lafu/motB, lafW, motA, motA, motB, motX, motY, } \\
\hline \multicolumn{4}{|l|}{ motY, scrA, scrB, scrC, scrG, B565_1123 } \\
\hline \multicolumn{4}{|l|}{ Regulation } \\
\hline luxS, adeG & $\mathrm{Al}-2$ & ।, ॥ & Vibrio, Acinetobacter \\
\hline $\operatorname{cas} A$ & CAl-1 & $\|$ & Vibrio \\
\hline \multicolumn{4}{|l|}{ Secretion system } \\
\hline epsA, epsB, epsC, epsE, epsF, epsG, epsH, epsl, epsJ, & EPS T2SS & । & Vibrio \\
\hline \multicolumn{4}{|l|}{ epsK, epsL, epsM, eps $N, g s p D$} \\
\hline exsA, exsD, sycN, tyeA, vcrD, vcrG, vcrH, vcrR, vcrV, & T3SS1 & I & Vibrio \\
\hline \multicolumn{4}{|l|}{$\operatorname{vec} A$, virG, vopB, vopD, vopN, vopQ, vopR, vscB, vscC, } \\
\hline \multicolumn{4}{|l|}{$v s c D$, vscF, vscG, vscH, vscl, vsCJ, vscK, vsCL, vscN, } \\
\hline$v s c O, v s c Q, v s c R, v s c S, v s c T, v s X U, v s c X, v s c Y, v s c C$ & & & \\
\hline hopAJ2, hopJ1, m/r6326, ati2, ati1 & Other T3SS & I, II & Pseudomonas, Mesorhizobium, Aerom \\
\hline
\end{tabular}


Table 3 Virulence factors predicted with VFDB database (Continued)

\begin{tabular}{|c|c|c|c|}
\hline Virulence factor & Annotation & Chromosome & Identity genus \\
\hline Ipg2936, trwD, virB1, virB10, CbuK_1823, CbuG_0575, & T4SS & I, II & Legionella, Bartonella, Brucella, Coxiella \\
\hline \multicolumn{4}{|l|}{ CbuG_1738, COXBURSA331_A0369 } \\
\hline PFLU6009, Psyr_2628, clpV1, pppA, clpV1, Psyr_2628, & T6SS & I, II & Pseudomonas, Burkholderia, Citrobacter, \\
\hline$t s s H / c l p V / b s c F, c t s 2 A$, lip2, impB, impB, impC, impC & & & Agrobacterium \\
\hline \multicolumn{4}{|l|}{ Toxin } \\
\hline $\sec A 2$ & Ace & I & Mycobacterium \\
\hline th & $\begin{array}{l}\text { Thermolabile } \\
\text { hemolysin }\end{array}$ & ॥ & Vibrio (Vibrio parahaemolyticus) \\
\hline$c|b Q, c| b M, c|b L, c| b|, c| b H, c|b F, c| b E, c|b D, c| b C$ & Colibactin & I & Escherichia \\
\hline vctA & Enterobactin & $\|$ & Vibrio \\
\hline ast & Enterotoxin & I & Aeromonas \\
\hline \multicolumn{4}{|l|}{ Immune evasion } \\
\hline ABK1_0097, ABK1_0097, BC5270, BC5275, BC5275, & Capsule & I, II & Vibrio, Staphylococcus, Mycoplasma, \\
\hline BCE_5384, cap8J, cpsA, cpsB, cpsB, cpsC, cpsD, cpsE, & & & Enterococcus, Acinetobacter, Neisseria, \\
\hline cpsF, cpsG, cpsJ, eps9, eps9, kpsF, kpsF, oppF, oppF, & & & Enterococcus, Campylobacter, Burkholderia, \\
\hline oppF, rm/B, rm/D, sicC/synD, uppS, wbfB, wbfV/wcvB, & & & Bacillus \\
\hline \multicolumn{4}{|l|}{$w c b N, w e c A, w z a, w z b, w z c$} \\
\hline fabZ, acpXL, kbtB, waaZ, waaF, gtrB & LPS & I & Brucella, Helicobacter, Pseudomonas, Shigella \\
\hline ACICU_00876, ASA_0824, ASA_3329, cbsB, ccmA, ccmB, & Iron uptake & I, II & Vibrio, Acinetobacter, Aeromonas, \\
\hline ccmC,ccmE, ccmF, ccmF, chuY, ciud, fur, hemA, hemB, & & & Burkholderia, Dickeya, Escherichia, \\
\hline hem $C$, hemE, hemG, hemH, hemL, hemN, hemN, hitA, hitB, & & & Haemophilus, Legionella, Mycobacterium, \\
\hline hitC, hitC, hmuU, hutA, huvB, huvC, huvD, huvX, huvZ, & & & Pseudomonas, Salmonella, Shigella, \\
\hline iutA, mbtl, orbG, orbH, pchH, phuV, PSEEN2499, psuA, pvdH, & & & Yersinia \\
\hline \multicolumn{4}{|l|}{ pvsA, pvsB, pvsC, pvsD, pvsE, pvuA, pvuB, pvuC, pvuD, } \\
\hline \multicolumn{4}{|l|}{ pvuE, sitA, sitB, sitC, sitD, vabC, vctC, vctC, vctD, vctD, } \\
\hline \multicolumn{4}{|l|}{$v c t G, v c t G, v c t P, v i u C$} \\
\hline \multicolumn{4}{|l|}{ Antiphagocytosis } \\
\hline $\operatorname{alg} B, \operatorname{alg} W, \operatorname{alg} W, \operatorname{alg} U$, mucP, alg $R$ & Alginate & I, II & Pseudomonas \\
\hline pgi, mrsAvglmM & Exopolysaccharide & I & Haemophilus \\
\hline hasD, hasB, hasF & $\begin{array}{l}\text { Hyaluronic acid } \\
\text { capsule }\end{array}$ & I, II & Pseudomonas, Serratia, Yersinia \\
\hline katA, katA, clpC, clpE, clpP, ctpV, ibpA, msrA/B (pilB), & Stress protein & I, II & Neisseria, Listeria, Mycobacterium, \\
\hline $\operatorname{reg} X 3$, regXX, sodB, sodB, sodC1 & & & Legionella, Salmonella \\
\hline \multicolumn{4}{|l|}{ Exoenzyme } \\
\hline icl & Isocitratase & I & Mycobacterium \\
\hline mip, mip & Mip & I, II & Legionella \\
\hline Regulation & & & Mycobacterium, Legionella, Salmonella \\
\hline mprA, mprA, mprA, relA, rpoS, csrA, sigA & Regulation & I & \\
\hline \multicolumn{4}{|l|}{ Efflux pump } \\
\hline$m t r D$ & MtrCDE & । & Neisseria \\
\hline panc & Metabolic adaptation & & Mycobacterium \\
\hline \multicolumn{4}{|l|}{ Others } \\
\hline csgD, csgD, glnA1, ML1683, ML1683, leuD, MGA_1142, & Others & & \\
\hline ipaH, ECS88_3547, ndk, pdhB, ppsE, cysC1, argK, eno, plr/gapA, & & I, II & Vibrio, Acinetobacter, Escherichia, \\
\hline $\begin{array}{l}\text { hpt, sugC, sugC, sugC, sugC, sugC, trpD, bfmR, bfmR, bfmR, } \\
\text { bfmR, }\end{array}$ & & & Listeria, Mycobacterium, Mycoplasma, \\
\hline \multirow[t]{2}{*}{ vibD, vibE, vibA, WA1298, WA1298, WA1298, vctP } & & & Pasteurella, Pseudomonas, Salmonella, \\
\hline & & & Shigella, Streptococcus \\
\hline
\end{tabular}


Table 4 Antimicrobial resistance genes predicted with the ARDB database

\begin{tabular}{|c|c|c|c|c|}
\hline Gene_id & Identity (\%) & Resistance_Type & Antibiotic_Resistance & Description \\
\hline CU052_02205 & 47.53 & pbp2 & penicillin & penicillin-binding protein 2 \\
\hline CU052_02455 & 99.35 & tet34 & tetracycline & $\begin{array}{l}\text { oxytetracycline resistance phosphoribosyltransferase } \\
\text { domain-containing protein Tet(34) }\end{array}$ \\
\hline CU052_03895 & 47.65 & tolc & $\begin{array}{l}\text { aminoglycoside, glycylcycline, } \\
\text { macrolide, beta_lactam,acriflavin }\end{array}$ & outer membrane channel protein TolC \\
\hline CU052_04185 & 67.79 & ksga & kasugamycin & $\begin{array}{l}\text { 16S rRNA (adenine(1518)-N(6)/adenine(1519)-N(6))- } \\
\text { dimethyltransferase RsmA }\end{array}$ \\
\hline CU052_04200 & 40.24 & dfra26 & trimethoprim & type 3 dihydrofolate reductase \\
\hline CU052_05755 & 49.16 & mexw & - & multidrug efflux RND transporter permease subunit \\
\hline CU052_07910 & 46.51 & pbpla & penicillin & PBP1A family penicillin-binding protein \\
\hline CU052_09355 & 42.63 & pbp1b & penicillin & penicillin-binding protein 1B \\
\hline CU052_09890 & 49.11 & pbp2 & penicillin & penicillin-binding protein 2 \\
\hline CU052_13950 & 51.93 & adeb & chloramphenicol, aminoglycoside & multidrug efflux RND transporter permease subunit \\
\hline CU052_15205 & 98.25 & norm & $\begin{array}{l}\text { tigecycline, streptomycin, kanamycin, } \\
\text { ciprofloxacin, norfloxacin }\end{array}$ & MATE family efflux transporter \\
\hline CU052_15655 & 43.59 & emre & aminoglycoside & $\begin{array}{l}\text { QacE family quaternary ammonium compound efflux } \\
\text { SMR transporter }\end{array}$ \\
\hline CU052_16225 & 48.04 & $b c r$ & - & $\mathrm{Bcr} / \mathrm{CflA}$ family drug resistance efflux transporter \\
\hline CU052_21770 & 40.74 & $y k k c$ & na_antimicrobials & $\begin{array}{l}\text { QacE family quaternary ammonium compound efflux } \\
\text { SMR transporter }\end{array}$ \\
\hline CU052_23115 & 43.06 & ceob & chloramphenicol & multidrug efflux RND transporter permease subunit \\
\hline CU052_25305 & 44.41 & emrd & - & multidrug transporter EmrD \\
\hline CU052_26580 & 43.94 & catb5 & chloramphenicol & antibiotic acetyltransferase \\
\hline CU052_27055 & 58.33 & qnra & fluoroquinolone & Qnr family pentapeptide repeat protein \\
\hline CU052_27855 & 62.23 & $m d t$ & chloramphenicol & multidrug transporter MdtL \\
\hline CU052_28095 & 100 & tetm & tetracycline & $\begin{array}{l}\text { tetracycline resistance ribosomal protection protein } \\
\text { Tet(M) }\end{array}$ \\
\hline CU052_28120 & 100 & tetb & tetracycline & tetracycline efflux MFS transporter Tet(B) \\
\hline CU052_28525 & 67.43 & anrs & fluoroquinolone & quinolone resistance pentapeptide repeat protein QnrVC6 \\
\hline CU052_28540 & 64.47 & dfra17 & Trimethoprim & trimethoprim-resistant dihydrofolate reductase DfrA \\
\hline CU052_29140 & 100 & sul2 & sulfonamide & sulfonamide-resistant dihydropteroate synthase Sul2 \\
\hline
\end{tabular}

(Fig. 6c and f). CU052_14535 was close to an outer membrane beta-barrel protein in $V$. splendidus (97.04\% identity), and an OmpA family protein in $V$. crassostreae ( $97.04 \%$ identity), suggesting recent acquisition of this gene from $V$. splendidus or $V$. crassostreae (Fig. 6c and f).

\section{HGT candidates on plasmids}

Five antibiotic resistance genes and one virulence gene, CU052_28670, involved in immune evasion were detected on plasmid p345-185. Basic local alignment search tool (BLAST) of nucleotide (Blastn) results showed high homo$\log$ (query cover $>40 \%$ and identity $>40 \%$ ) of p345-185 with plasmids pVPS62, V36, pVPS114, pVPH2, and pVPS91 from $V$. parahaemolyticus; pVAS114 and pVAS19 from $V$. alginolyticus; and pAQU1 from Photobacterium damselae, but no homology with other $V$. harveyi plasmids
(Fig. 7a). Plasmid p345-185 showed 99.97\% homolog (query cover $=99 \%$ ) with pVPS62. CU052_29140 (sul2) was not found on 345 chromosomes and showed 100\% amino acid identity to sul2 in Acinetobacter baumannii, Escherichia coli, Actinobacillus pleuropneumoniae, Histophilus somnim, and Salmonella enteric (Fig. 7b).

\section{Nucleotide sequence accession numbers}

The complete genome sequence of $V$. harveyi 345 was deposited in GenBank with accession numbers CP025537 (ChI), CP025538 (ChII), CP025539 (plasmid p345-185) and CP025540 (plasmid p345-67).

\section{Discussion}

$V$. harveyi 345 was isolated and studied because of its multidrug resistance and serious virulence to $E$. oanceolutus in Shenzhen, Southern China. The complete genome 


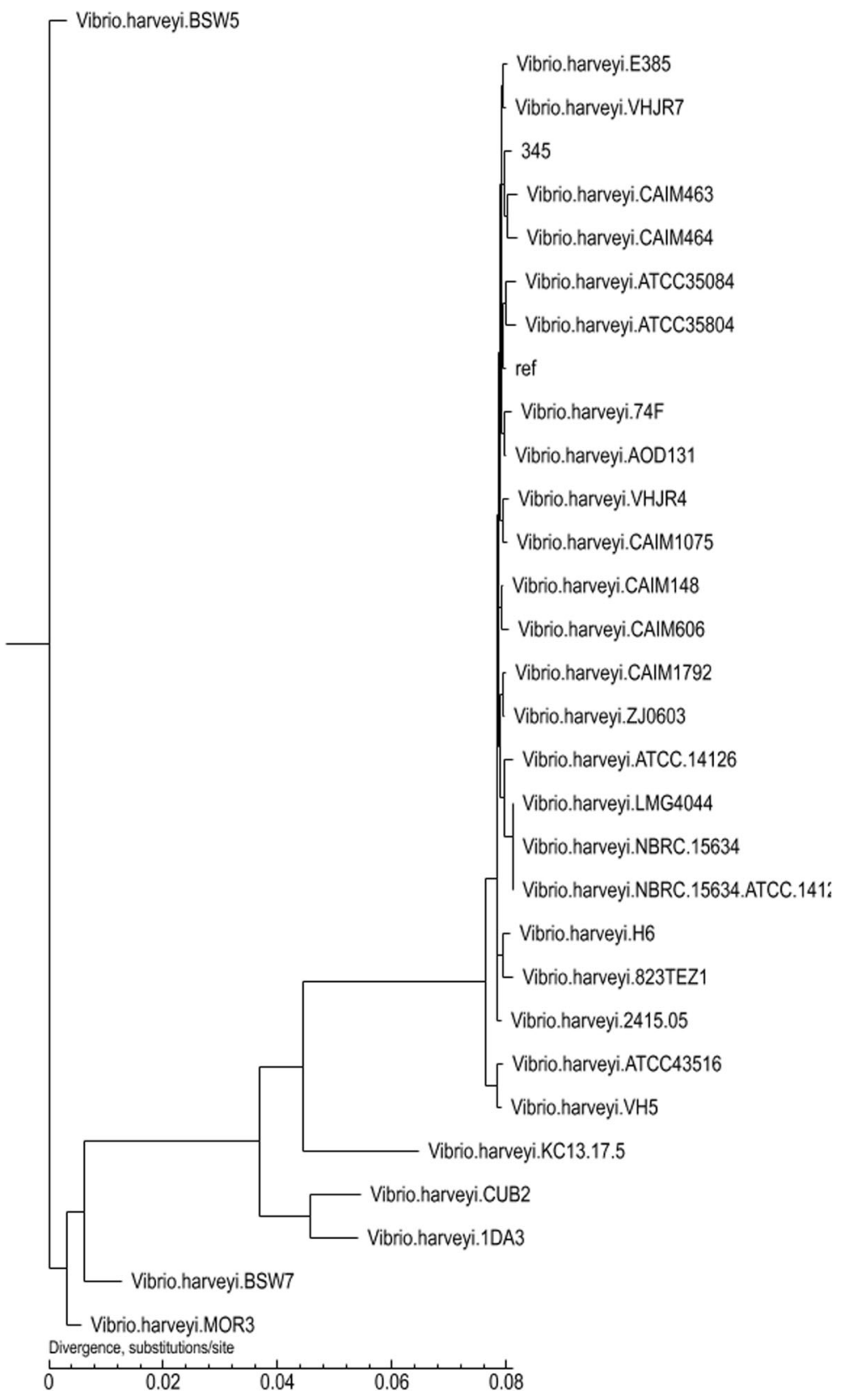

Fig. 3 Phylogenetic relationship of $V$. harveyi 345 and 30 compared $V$. harveyi strains. Results are based on the SNP matrix of the 31 strains and Treebest-1.9.2 software and maximum-likelihood method

sequence of $V$. harveyi 345 was determined and compared with $30 \mathrm{~V}$. harveyi strains. $\mathrm{V}$. harveyi 345 was assembled into two circular chromosomes and two plasmids. It had a larger genome than 27 strains other than KC13.17.5, E385, and 74F and had a consistent $\mathrm{G}+\mathrm{C}$ content with the 30 others strains. Compared to the $30 \mathrm{~V}$. harveyi strains, $V$. harveyi 345 had more predicated genes than the others strains except for ZJ0603. In total, 487 virulence genes encoding proteins in flagella, iron uptake, pili, LPS, CPS, chemotaxis and type II/III/IV/VI secretion involved in fish disease in clinics, were identified in $V$. harveyi 345. In addition, 25 ARGs including genes for resistance to aminoglycoside (golc, adeb, emre), penicillin ( $p b p 2$, pbp1a, $p b p 1 b, p b p 2)$, tetracycline (tet34, tetm, tetb), chloramphenicol (adeb, ceob, $m d t l$ ) and trimethoprim (dfra26) were found in $V$. harveyi 345 , consistent with its resistance to streptomycin, ampicillin, tetracycline, chloramphenicol, trimethoprim, and sulfamethoxazole.

Virulence genes and antimicrobial-resistance genes are acquired by bacterial replication and by HGT. Horizontal 


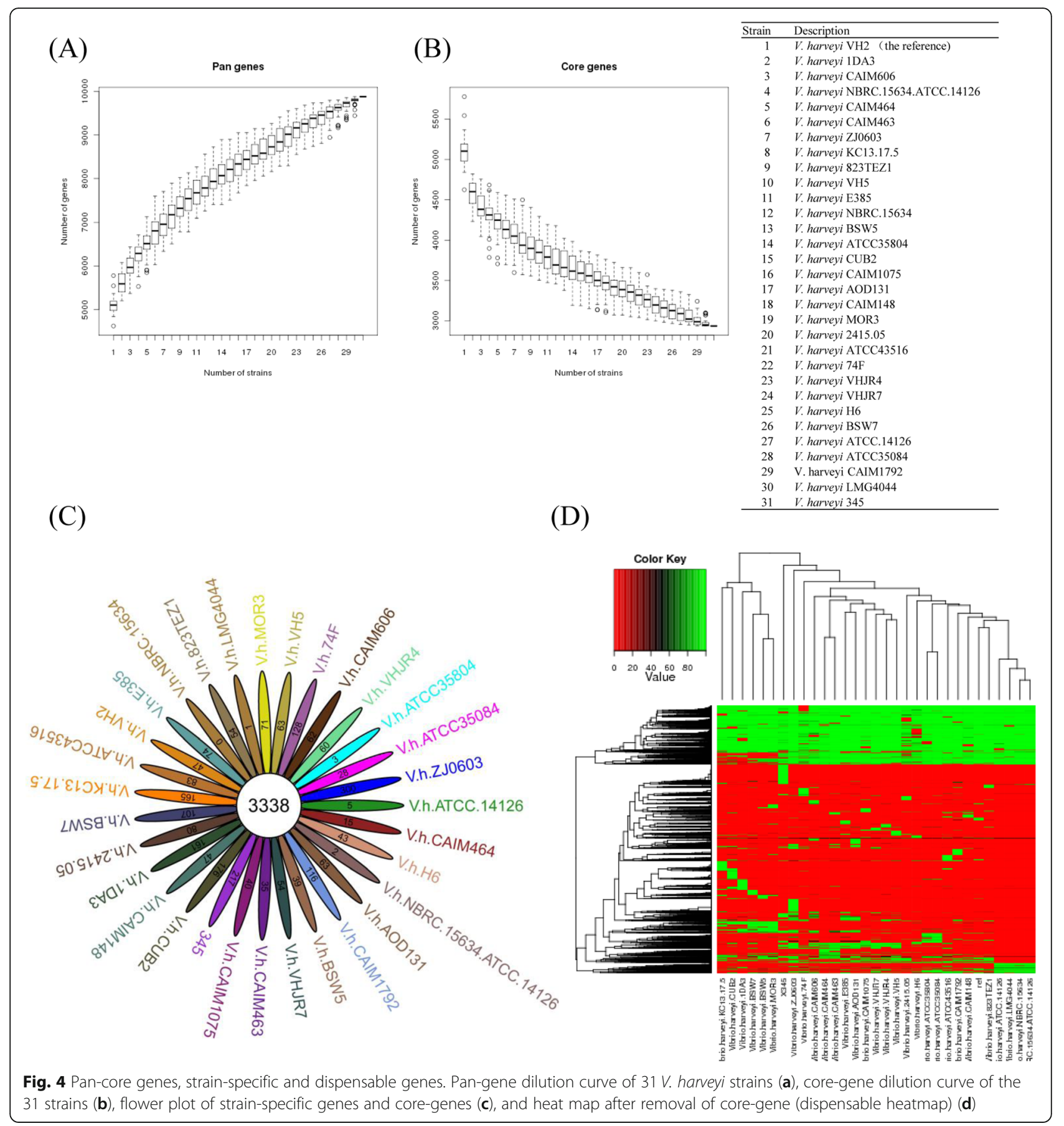

transfer is the exchange of genetic material within species without any sexual mechanism [43]. This phenomenon is widely documented in bacteria and has a role in bacterial evolution and adaptation [43]. Comparative genomics of $31 \mathrm{~V}$. harveyi strains was conducted to analyze HGT events by identifying strain-specific genes and strain-gene families. Considering the average gene number of 5087 for the $31 \mathrm{~V}$. harveyi strains, the 3338 core genes represented approximately $66 \%$ of the total genome, meaning that approximately two-thirds of the genome was conserved among all strains. However, flower plots and dispensable heatmaps showed that each stain contained strain-specific genes, probably obtained by HGT. A total of 217 genes were specific to $V$. harveyi 345 , which was more than other strains except for ZJ0603. This result suggested that a large number of HGT events happened in 345. In addition, seven gene families were specific to $V$. harveyi 345 . Most $(80.65 \%$, $175 / 217$ ) of the strain-specific genes had $>40 \%$ identify to other Vibrio species. The remaining came from other genera such as Shewanella, Photobacterium, Pseudovibrio, and 

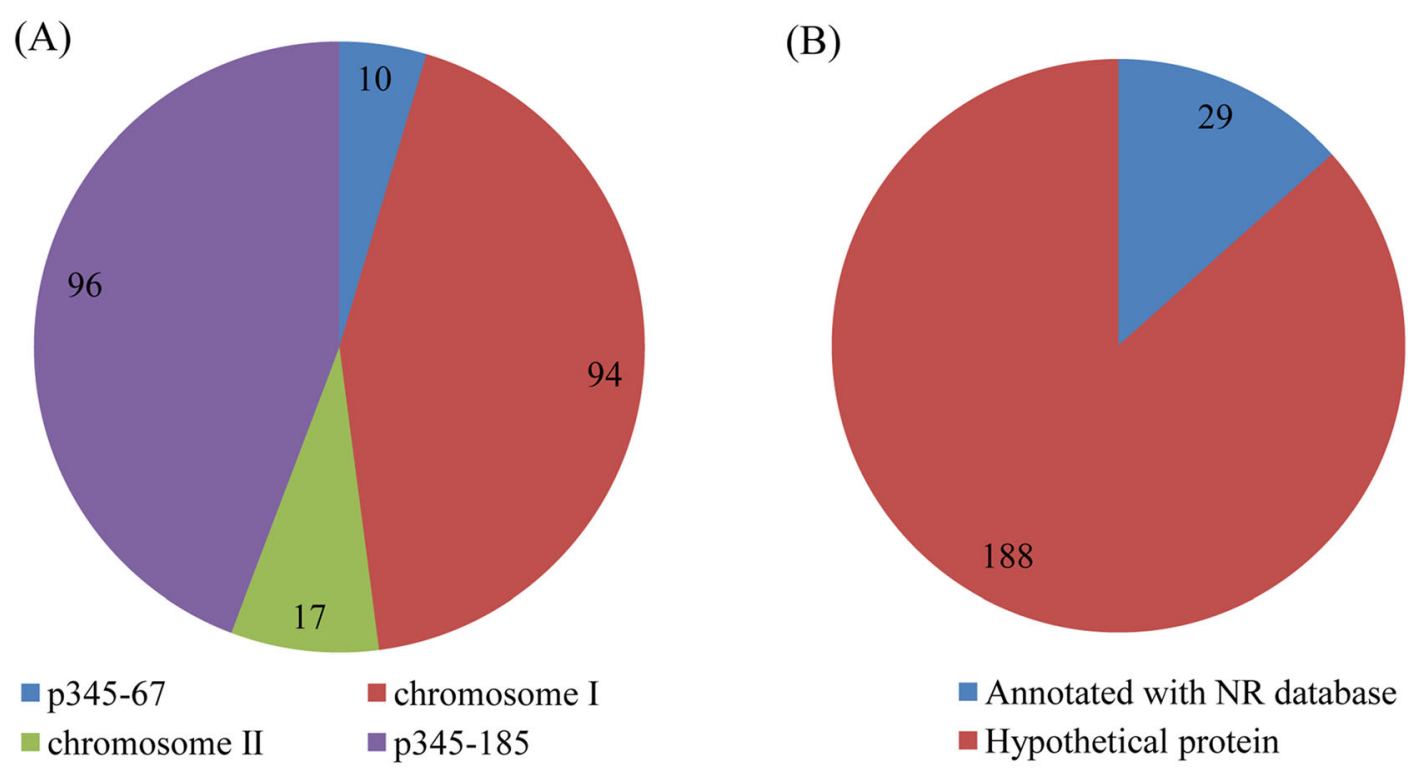

- Annotated with NR database - Hypothetical protein

(C)

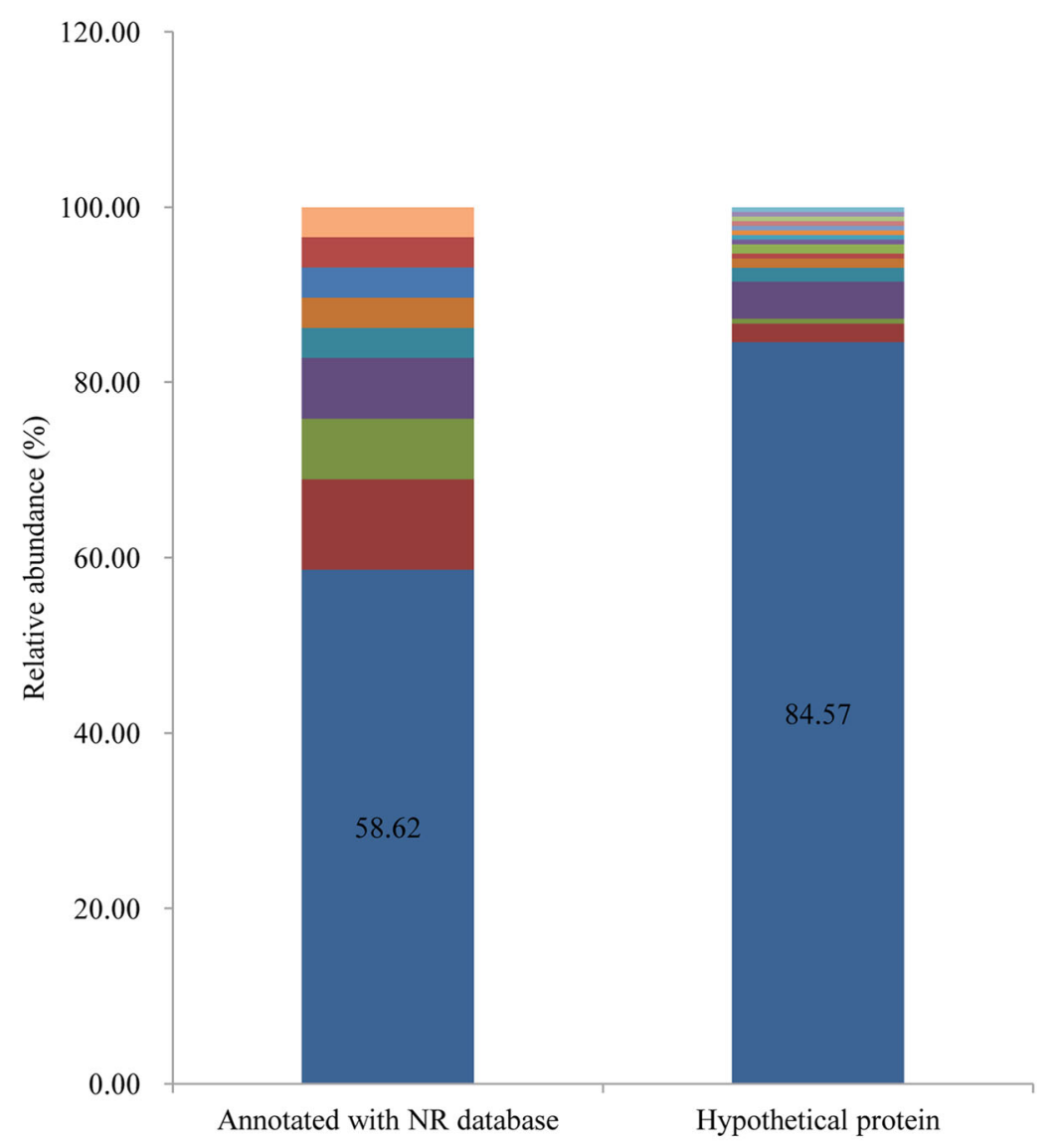

$\square$ uncultured bacterium

- Endozoicomonas spp.

- Yersinia spp.

- Oxalobacter spp.

- Yersinia spp.

- Xenorhabdus spp.

- Proteus spp.

- Arsukibacterium spp.

- Achromobacter spp.

- Gammaproteobacteria spp.

- Morganella spp.

- Providencia spp.

- Escherichia spp.

- Pseudovibrio spp.

- Photobacterium spp.

- Shewanella spp.

- Burkholderia spp.

Vibrio spp.

Fig. 5 Strain-specific genes of $V$. harveyi 345. Strain-specific genes locations (a). Gene number of hypothetical proteins and proteins annotated by NR Database (b). Composition and relative abundance of strain-specific genes (c) 
Table 5 Strain-specific gene families of $V$. harveyi 345

\begin{tabular}{|c|c|c|c|c|c|}
\hline Gene Family ID & Locus & Identity & E_value & Subject ID & Subject_description \\
\hline 1 & CU052_06110 & 91.98 & $1.00 \mathrm{E}-125$ & gi|752,301,544|ref.|WP_041157164.1| & hypothetical protein, partial [Vibrio mytill] \\
\hline 1 & CU052_29120 & 100 & $0.00 E+00$ & gi|446,775,679|ref.|WP_000852935.1| & recombinase [Escherichia coli] \\
\hline 2 & CU052_00970 & 62 & $0.00 E+00$ & gi|359,344,462|gb|AEV37836.1| & $\begin{array}{l}\text { Putative peptide synthetase [Pseudovibrio sp. } \\
\text { FO-BEG1] }\end{array}$ \\
\hline 2 & CU052_00980 & 55.33 & $0.00 \mathrm{E}+00$ & gi|504,051,870|ref.|WP_014285864.1| & $\begin{array}{l}\text { non-ribosomal peptide synthetase [Pseudovibrio } \\
\text { sp. FO-BEG1] }\end{array}$ \\
\hline 3 & CU052_00975 & 61.49 & $2.00 \mathrm{E}-125$ & gi|504,051,870|ref.|WP_014285864.1| & $\begin{array}{l}\text { non-ribosomal peptide synthetase [Pseudovibrio } \\
\text { sp. FO-BEG1] }\end{array}$ \\
\hline 3 & CU052_00965 & 62.42 & $0.00 \mathrm{E}+00$ & gi|359,344,462|gb|AEV37836.1| & $\begin{array}{l}\text { Putative peptide synthetase [Pseudovibrio sp. } \\
\text { FO-BEG1] }\end{array}$ \\
\hline 4 & CU052_28750 & 100 & 8.00E-84 & gi|760,459,713|gb|AJP18243.1| & $\begin{array}{l}\text { MULTISPECIES: type IV conjugative transfer system } \\
\text { protein TraA [Vibrio parahaemolyticus] }\end{array}$ \\
\hline 4 & CU052_28755 & 100 & $3.00 \mathrm{E}-84$ & gi|691,549,048|ref.|WP_032073022.1| & hypothetical protein [Vibrio sp. 04Ya090] \\
\hline 5 & CU052_05575 & 75.81 & $6.00 \mathrm{E}-93$ & gi|647,339,569|ref.|WP_025768281.1| & integrase [Vibrio parahaemolyticus] \\
\hline 5 & CU052_29060 & 100 & $2.00 \mathrm{E}-107$ & gi|504,199,683|ref.|WP_014386785.1| & hypothetical protein [Photobacterium damselae] \\
\hline 6 & CU052_00925 & 50.69 & $0.00 E+00$ & gi|359,344,454|gb|AEV37828.1| & Polyketide synthase [Pseudovibrio sp. FO-BEG1] \\
\hline 6 & CU052_01010 & 56.65 & $0.00 E+00$ & gi|359,344,469|gb|AEV37843.1| & Polyketide synthase [Pseudovibrio sp. FO-BEG1] \\
\hline 7 & CU052_28405 & 96.77 & $2.00 \mathrm{E}-80$ & gi|504,199,762|ref.|WP_014386864.1| & hypothetical protein [Photobacterium damselae] \\
\hline 7 & CU052_28260 & 100 & $6.00 \mathrm{E}-96$ & gi|504,199,733|ref.|WP_014386835.1| & hypothetical protein [Photobacterium damselae] \\
\hline
\end{tabular}

Escherichia. We focused on the characterization of three strain-specific genes, CU052_00920, CU052_13320, and CU052_14535. CU052_00920 encoded a class C betalactamase and was transferred from Pseudovibrio spp. Betalactamase enzymes are reported to inactivate beta-lactam antibiotics by hydrolyzing the peptide bond of the characteristic four-membered beta-lactam ring, rendering the antibiotic ineffective [44]. This result suggested that CU052_00920 contributed to antibiotic resistance, especially ampicillin resistance of $V$. harveyi 345. CU052_13320 encoded a virulence-associated protein $\mathrm{D}$ and closes to a virulence factor in Pseudomonas aeruginosa (73.40\% identity) and Aeromonas sobria (75.53\% identity). CU052 13320 probably contributed to the virulence of 345 . CU052_14535 encoded an OmpA family protein and was acquired from $V$. splendidus or $V$. crassostreae. Among pathogenic bacteria, OmpA proteins are important for pathogenesis including bacterial adhesion, invasion, or intracellular survival and evasion of host defenses or stimulation of proinflammatory cytokine production [45]. This result suggested that CU052_14535 regulates the virulence of $V$. harveyi 345 . DNA can also be horizontally transmitted between bacteria through plasmids, phages or uptake of naked DNA from environment [46]. Homology analysis showed that p345-185 had 99.97\% homology (query cover $=99 \%$ ) to pVPS62 in $V$. parahaemolyticus. PVPS62 is reported to be a pAQU-type plasmid and emerge MDR conjugative plasmid among important pathogens [47]. Five antimicrobial-resistance genes (tetm, tetb, qurs, dfra17, and sul2) involved in resistance to tetracycline, fluoroquinolone, trimethoprim, and sulfonamide, and one virulence gene (CU052_28670) involved in immune evasion were located on plasmid p345-185. These genes could be horizontally transferred, promoting drug resistance and virulence. Especially, CU052_29140 (sul2) showing 100\% amino acid identity to sul2 in Acinetobacter baumannii, Escherichia coli, Actinobacillus pleuropneumoniae, Histophilus somnim, and Salmonella enteric, should contribute to the resistance of pediatric compound sulfamethoxazole tablets and sulfamethoxazole. Similarly, Klein et al. [48] found that chloramphenicol, oxytetracycline and chlortetracycline could be successful transferred by R-plasmids.

Except for plasmids, HGT encompasses a variety of genetic units, collectively known as MGEs [49] and including phages, GIs, and integrating conjugative elements (ICEs). A total of 47 GIs, with encoding many transposases, integrases, and recombinases, and two incomplete prophage sequences were identified in $V$. harveyi 345. These genetic units probably acted as HGT delivery tools, contributing to pathogenesis, drug resistance and environmental adaptation of $V$. harveyi 345. Several type III/VI secretion proteins along with IS family transposases were predicted on the same GI that could be easily transmitted. Dissemination of these genes could further compromise Vibrio infections, limiting treatment options. These results further indicated that HGT contributed to virulence and antibiotic resistance of $V$. harveyi 345.

The 345 strain was isolated from a diseased E. oanceolutus in Shenzhen, Southern China. Recent increasing 
A

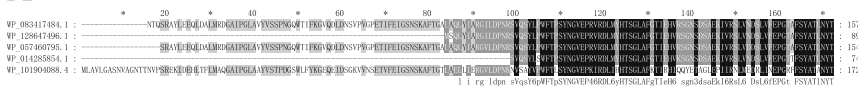

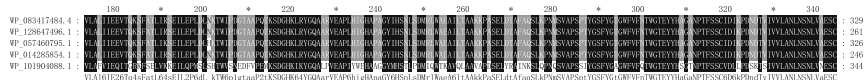

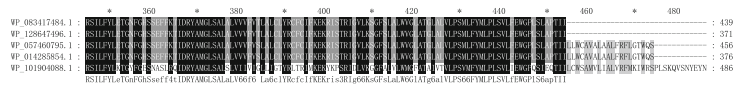

B

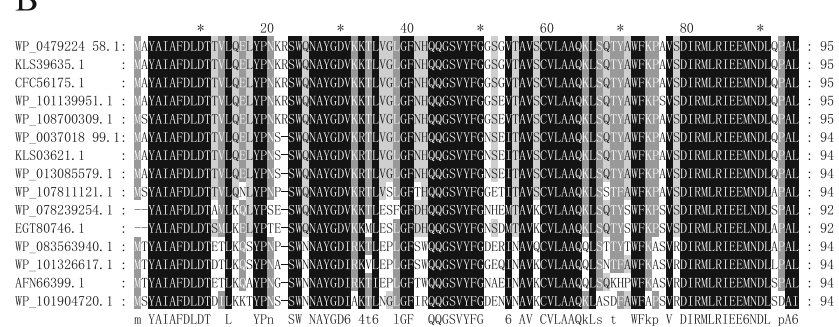

C
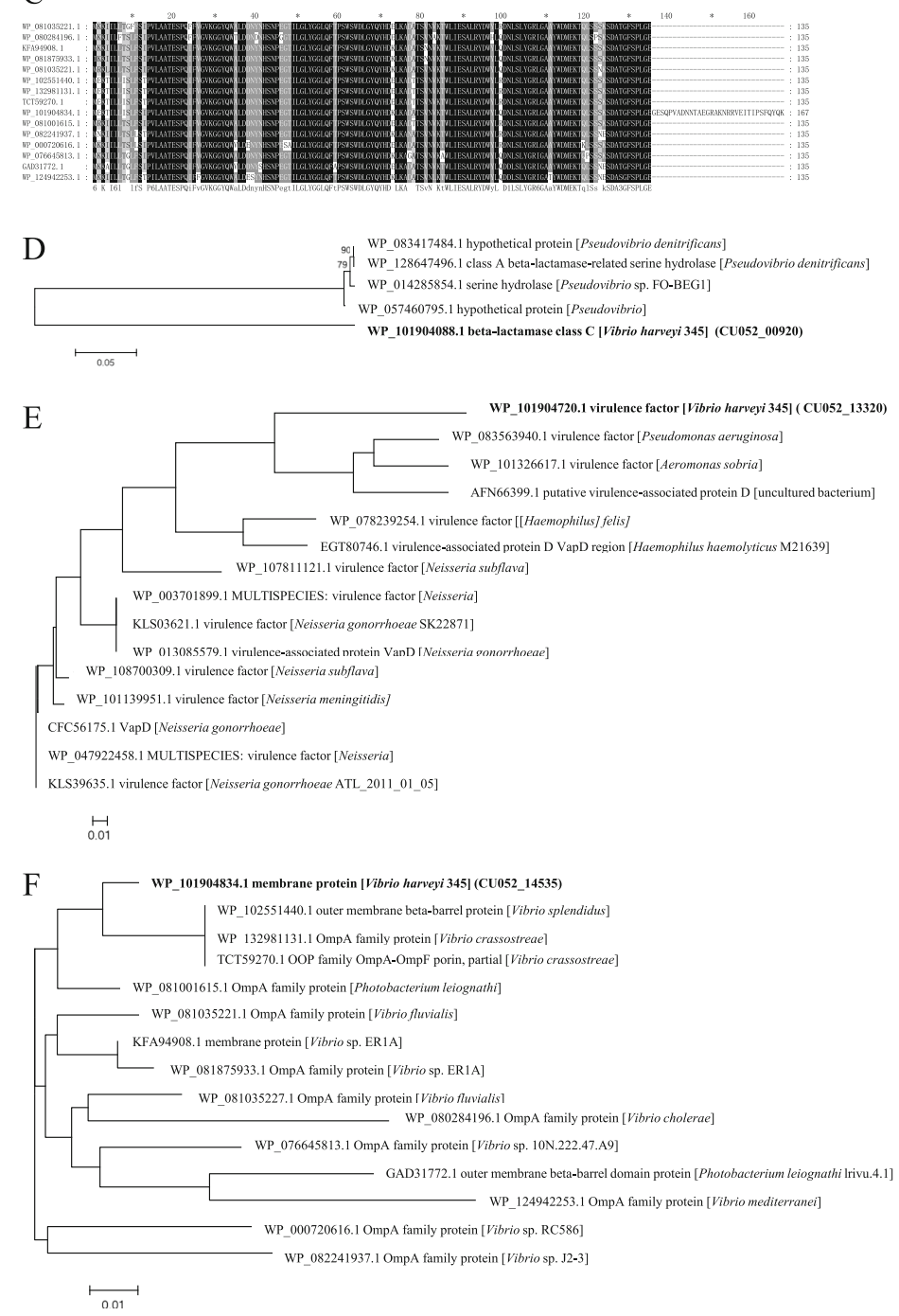

Fig. 6 Evolutionary analysis of CU052_00920, CU052_13320, and CU052_14535. Multisequence alignment (a-c) and phylogenetic analysis (D-F) of CU052_00920, CU052_13320, and CU052_14535 with amino acids. Multisequence alignment was by ClustalW and phylogenetic tree was constructed with MEGA 6. Bar substitutions per sequence position: 0.05 (d), $0.01(\mathbf{e})$, and $0.01(\mathbf{f})$ 


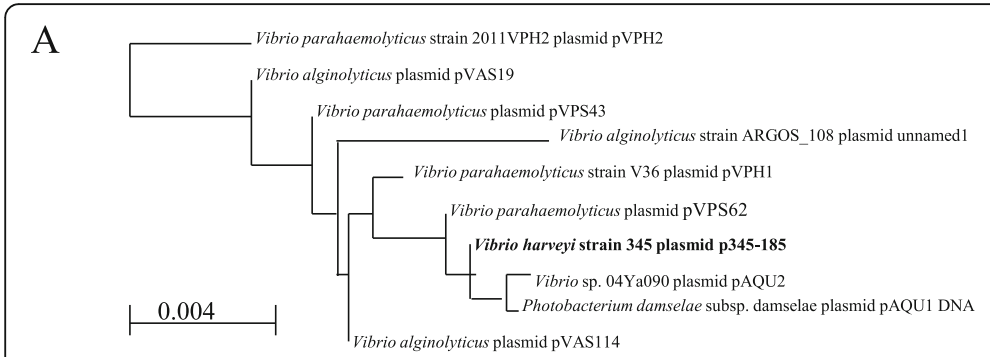

B
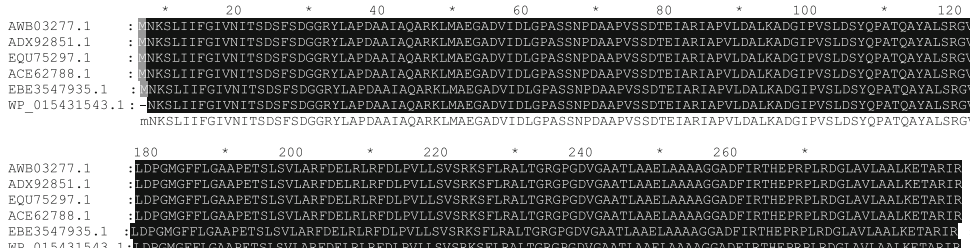

Fig. 7 Evolutionary analysis of p345-185 and CU052_29140. Nucletides phylogenetic analysis of p345-185 (a) and amino acids multisequence alignment of CU052_29140 (b). Phylogenetic tree was constructed with the neighbor-joining method, max seq difference $=0.75$ using BLAST pairwise alignments. Multisequence alignment was by ClustalW

aquaculture density has led to frequent breeding diseases and increased use of antibiotics in Southern China, resulting in serious antibiotic residues and pollution [50]. Intensification of coastal urbanization has led to massive discharge of pollutants (heavy metals, nutrients, biocides), and intensification of human activities has caused environmental climate change, especially global warming [51]. Many studies show that the wide use of antibiotics; pollution by heavy metals, nutrients and biocides; and global climate change regulate HGT by affecting plasmid replication, changing phage activity, adjusting HGT-related enzyme activity, and damaging immune systems. These factors regulate bacterial resistance and pathogenicity [52-54]. For example, Beaber et al. [53] found that ciprofloxacin induces transfer of SXT, an ICE derived from $V$. cholera encoding genes that confer resistance to chloramphenicol, sulphamethoxazole, trimethoprim and streptomycin. The mechanism is increasing expression of SXT activators, enhancing drug resistance. Similarly, high environmental temperatures in combination with UV irradiation accelerate the spread of stx (Shiga toxin) genes by enhancing Stx prophage induction and Stx phagemediated gene transfer [52]. Therefore, environmental pollution and climate change may increase the communication of virulence genes and drug resistance genes by affecting HGT. The result will enhance the toxicity and drug resistance of $V$. harveyi and affect the ecological safety of aquaculture ecosystems.

\section{Conclusions}

We present the first complete genome of the serious disease-causing $V$. harveyi 345 which has a multidrugresistant phenotype. We did comparative genomics between this strain and 30 other $V$. harveyi strains. The genome was determined to study $V$. harveyi 345 virulence and antimicrobial resistance. Multiple virulence factors and resistance genes were predicted in this strain, consistent with results of Jiang et al. [38]. We searched for evidence of HGT and evaluated genomic traits relating to HGT. The high quality complete genome sequence generated in this study will aid further studies for a deeper understanding of the mechanisms of Vibrio pathogenesis and antibiotic resistance. These studies could improve seafood quality and reduce economic loss. We recommend that more research be done on the response mechanism of HGT relative to environmental change and antibiotic use. This research will be an important scientific basis for predicting outbreaks and controlling $V$. harveyi disease. As environmental pollution including use of antibiotics and climate change enhance the virulence and drug resistance of pathogens, research should explore management approaches, for example by bacteriophages, to manage the occurrence of $V$. haveyi in the environment [55].

\section{Methods \\ DNA extraction}

$V$. harveyi 345 was grown overnight in 2216E medium (BD, USA) at $28^{\circ} \mathrm{C}$ with vigorous shaking. Overnight cultures were inoculated with a 1:1000 dilution into 20 $\mathrm{ml}$ fresh 2216E medium and grown until OD600 $=0.5$. Cells were collected by centrifugation and genomic DNA extracted using the CTAB method [56]. The quantity and quality of genomic DNA were evaluated using a Qubit Fluorometer (Invitrogen, Carlsbad, CA, USA) and $1 \%$ agarose gel electrophoresis. DNA was stored at $80^{\circ} \mathrm{C}$ and prepared for genome sequencing. 
Whole genome sequencing and assembly

A PacBio RSII 20-kb template library was constructed from at least $10 \mu \mathrm{g}$ genomic DNA at the Beijing Genomics Institute (BGI). The library was subjected to quality control (QC) with Qubit Fluorometer (Invitrogen, Carlsbad, CA, USA) and Nanodrop 2000 (Thermo Fisher Scientific, Wilmington, DE, USA) to check the concentration, purity and integrity of library templates. Libraries were sequenced on Illumina Hiseq 4000 and Pacbio RSII sequencing platforms. Genomes were primarily assembled with a variety of software for short sequence assembly based on clean data from the Illumina Hiseq 4000 platform. RS HGAP Assembly3 of SMRT Analysis v.2.3.0 was used to assemble data from the Pacbio RSII platform. Contigs were error corrected successively by soapSNP and soapIndel software and GATK analysis. Gaps between contigs were filled by PCR.

\section{General annotation}

ORFs were predicted by Glimmer v.3.02 [57], and genome annotation was completed using the NCBI prokaryotic genome automatic annotation pipeline with identity $\geq 40 \%$ and E_value $\leq 10^{-5}$ (Blast and annotation for all study sets used the same identities and E_values). COG, KEGG, GO and NR databases were used to search domain architecture. The tRNA genes were identified by tRNAscan-SE v.1.23 [58] and rRNA genes with RNAmmer v.1.2 [59]. The sRNAs were predicted by blasting nucleic acid against Rfam database [60]. Tandem repeats were predicted by Tandem Repeat Finder v.4.04 [61].

\section{Identification of virulence factors and ARGs}

Putative virulence factors of $V$. harveyi 345 were predicted by blasting against the Virulence Factor Database (VFDB) (http://www.mgc.ac.cn/VFs/main.htm) [62] and ARGs by blasting against the Antibiotic Resistance Genes Database (ARDB) (http://ardb.cbcb.umd.edu/) [63].

\section{Prediction of Gls, prophages, and CRISPR-Cas systems}

GIs, prophages, and CRISPR-Cas systems were respectively identified using online tools IslandViewer 4, PHAge Search Tool (PHAST), and CRISPR Finder software [64-66].

\section{Comparative genomics}

Comparative genome analysis was conducted among $31 \mathrm{~V}$. harveyi strains with $V$. harveyi $\mathrm{VH} 2$ as the reference strain and an average gene number of 5087 (Additional file 2: Table S2) to assess $V$. harveyi evolution. A phylogenetic tree was constructed based on the single nucleotide polymorphism (SNP) matrix of the 31 strains with the maximum-likelihood method, bootstrapped 1000 times via Treebest-1.9.2 software [67].
Extraction of pan-, core-, dispensable and strain-specific genes

Core-pan genes were analyzed using a BLAST method [68]: Genes were taken from reference genome $V$. harveyi $\mathrm{VH} 2$ as a gene pool. Genes predicted in one strain among other 30 strains (Query samples) were BLAST with the gene pool, and results filtered by length and identity $(>40 \%)$. BLAST coverage ratios $(B C R$, reference $\mathrm{BCR}=$ match $/$ reference length $\times 100 \%$, query $\mathrm{BCR}=$ match/query length $\times 100 \%$, and match stands for the length used for BLAST) of genes from the gene pool and Query samples were calculated separately. If BCR values from the reference and Query sample were smaller than the setting value $(40 \%)$, the reference gene did not have homology with the Query gene. The nonhomologous gene from the Query genome was added to the gene pool. Query samples were processed and the final gene pool used as the pan gene pool. The non-homolog genes from the reference genome were the strain specific genes against the Query sample. Nonhomologous genes were BLAST with the gene pool of another Query sample, repeated for all samples, and the final nonhomologous gene pool was used as the strain-specific gene pool.

After aligning to genes from samples, BCR values of genes from the pan gene pool were calculated for each sample. The coverage array was generated for this pool. If the BCR value of a gene in each sample was larger than the setting value, the gene was a core gene. If the gene was predicted from an assembled result, BLAST results were filtered and the sequence was removed if the number $\mathrm{N}$ ( $\mathrm{N}$ means uncertain nucleotides in gene) was larger than the setting (30\%) for the gene. Dispensable genes were included in the pan-gene pool but not the core-gene pool.

\section{Construction of gene families}

Gene families were constructed using genes from the reference strain and the target bacterium. Protein sequences were aligned with basic local alignment search tool of protein (Blastp) and redundancy was eliminated. Gene family TreeFam clustering was carried out with alignment results and Hcluster_sg software. Alignment results for proteins were converted into multiple sequence amino acids in the CDS area after multiple sequence alignments with the clustered gene family using Muscle software [69]. Gene family tree construction analysis was carried out for multiple sequence alignment results using Muscle and neighborhood-joining (NJ) method with Treebest software [67].

\section{Characterization of putative HGT}

Of the strain-specific genes, we extracted CU052_00920, encoding a beta-lactamase class C; CU052_13320, encoding a virulence-associated protein $\mathrm{D}$; and CU052 
14535 encoding an OmpA family protein for further analysis on contribution to the antibiotic resistance or virulence of $V$. harveyi 345 . The genes were compared to the NR protein database of NCBI using the Blastp tool. The results were filtered manually to find significant hits to proteins belonging to species other than $V$. harveyi. Multiple sequence alignment was conducted using ClustalW [70]. An alignment phylogenetic tree was constructed from aligned sequences using the Kimura 2-parameter model [71] with the neighborjoining method, bootstrapped 1000 times, with MEGA6.0 software [72].

In addition, the entire sequence of p345-185 was compared to the NR nucleotide database of NCBI using a Blastn tool. A phylogenetic tree was constructed with the neighbor-joining method using max seq difference $=0.75$ and BLAST pairwise alignments. The characterization method was the same for CU052_29140 (sul2) on p345185 as for CU052_00920, CU052_13320, and CU052 14535.

\section{Supplementary information}

Supplementary information accompanies this paper at https://doi.org/10. 1186/s12864-019-6137-8

Additional file 1: Table S1. Global comparison of pollutant (antibiotics, heave metals, and nutrients) concentrations of water samples.

Additional file 2: Table S2. Gene information and gene statistics for $31 \mathrm{~V}$. harveyi strains.

Additional file 3: Figure S1. KEGG pathway classifications of $V$. harveyi 345 coding sequences.

Additional file 4: Figure S2. GO functional classifications of $V$. harveyi 345 coding sequences

Additional file 5: Table S3. Details of virulence factors predicted with the VFDB database.

Additional file 6: Table S4. Genomic islands in a single chromosome (CP025537) of $V$. harveyi 345.

Additional file 7: Table S5. Genomic islands in a single chromosome (CP025538) of $V$. harveyi 345.

Additional file 8: Table S6. Prophage sequences present in $V$. harveyi 345 chromosomes.

Additional file 9: Table S7. Strain-specific genes of $V$. harveyi 345 .

\section{Abbreviations}

ARDB: Antibiotic resistance genes database; ATG: Antibiotic-resistance gene; BGI: Beijing Genomics Institute; BLAST: Basic local alignment search tool; COG: Clusters of orthologous groups; Gls: Genetic islands; GO: Gene ontology; HGT: Horizontal gene transfer; ICEs: Integrative and conjugative elements; KEGG: Kyoto encyclopedia of genes and genomes; LD50: Median lethal dose; MDR: Multidrug resistance; MGE: Mobile genetic element; NJ: Neighborhood-joining; NR: Non-Redundant; ORFs: Open reading frames; PHAST: PHAge Search Tool; QC: Quality control; RM: restriction modification; SNP: Single nucleotide polymorphism; T6SS: Type VI secretion system; TLH: Thermolabile hemolysin; VFDB: Virulence factor database

\section{Acknowledgements}

We would like to thank International Science Editing (http://www. internationalscienceediting.com) for editing this manuscript.

\section{Authors' contributions}

$L X$ isolated the strain. YD, HX and JF designed the project. JW extracted the DNA. YD, YS and ZG performed the computational analyses. YD interpreted the data and wrote the manuscript. SL and CC critically revised the manuscript. All authors read and approved the final manuscript.

\section{Funding}

This work was supported by grants from the Hainan Provincial Natural Science Foundation of China (319QN336), the Natural Science Fund of Guangdong (2018A030310695), the National Natural Science Foundation of China (31902415, 41806147), Guangdong YangFan Innovative \& Entepreneurial Research Team Program (2016YT03H038), and the China Agriculture Research System (CARS-48). The funders had no role in study design, data collection and analysis, decision to publish, or preparation of the manuscript.

\section{Availability of data and materials}

Data generated or analysed during this study are included in this published article and its supplementary information files. Genomic data are available from the GenBank (accession numbers: CP025537, CP025538, CP025539 and (P025540).

Ethics approval and consent to participate

Not applicable.

\section{Consent for publication}

Not applicable.

\section{Competing interests}

The authors declare that they have no competing interests.

\section{Author details}

1Key Laboratory of South China Sea Fishery Resources Exploitation \& Utilization, Ministry of Agriculture and Rural Affairs, South China Sea Fisheries Research Institute, Chinese Academy of Fishery Sciences, Guangzhou 510300, China. ${ }^{2}$ Tropical Aquaculture Research and Development Centre, South China Sea Fisheries Research Institute, Chinese Academy of Fishery Sciences, Hainan 572426, China. ${ }^{3}$ Key Laboratory of Tropical Marine Bio-resources and Ecology, South China Sea Institute of Oceanology, Chinese Academy of Sciences, Guangzhou 510301, China.

Received: 8 August 2019 Accepted: 24 September 2019

Published online: 22 October 2019

\section{References}

1. Egidius E. Vibriosis: pathogenicity and pathology. A review. Aquaculture. 1987:67(1-2):15-28.

2. Osunla CA, Okoh Al. Vibrio pathogens: a public health concern in rural water resources in sub-Saharan Africa. Int J Environ Res Public Health. 2017; 14(10):1188.

3. Jiravanichpaisal P, Miyazaki T, Limsuwan C. Histopathology, biochemistry, and pathogenicity of Vibrio harveyi infecting black tiger prawn Penaeus monodon. J Aquat Anim Health. 1994;6(1):27-35.

4. Zorrilla I, Arijo S, Chabrillon M, Diaz P, Martinez-Manzanares E, Balebona MC, et al. Vibrio species isolated from diseased farmed sole, Solea senegalensis (Kaup), and evaluation of the potential virulence role of their extracellular products. J Fish Dis. 2003;26(2):103-8.

5. Karunasagar I, Pai R, Malathi GR, Karunasagar I. Mass mortality of Penaeus monodon larvae due to antibiotic-resistant Vibrio harveyi infection. Aquaculture. 1994;128(3-4):203-9.

6. Nishimori E, Hasegawa O, Numata T, Wakabayashi H. Vibrio carchariae causes mass mortalities in Japanese abalone, Sulculus diversicolor supratexta. Fish Pathol. 1998;33:495-502.

7. Luna GM, Bongiorni L, Gili C, Biavasco F, Danovaro R. Vibrio harveyi as a causative agent of the white syndrome in tropical stony corals. Environ Microbiol Rep. 2010;2(1):120-7.

8. Kraxberger-Beatty T, McGarey DJ, Grier HJ, Lin DV. Vibrio harveyi, an opportunistic pathogen of common Snook, Centropomus undecimalis (Bloch), held in captivity. J Fish Dis. 1990;13:557-60.

9. Saeed MO. Association of Vibrio harveyi with mortalities in cultured marine fish in Kuwait. Aquaculture. 1995;136(1-2):21-9. 
10. Company R, Sitj A, Bobadilla PMJ, Garay E, Alvarez-Pellitero P, et al. Bacterial and parasitic pathogens in cultured common dentex, Dentex dentex L. J Fish Dis. 1999;22(4):299-309.

11. Becker P, Gillan D, Lanterbeca D, Jangoux M, Rasolofonirina R, Rakotovao J, et al. The skin ulceration disease in cultivated juveniles of Holothuria scabra (Holothuroidea, Echinodermata). Aquaculture. 2004;242(1-4):13-30.

12. Ransangan J, Lal TM, Al-Harbi AH. Characterization and experimental infection of Vibrio harveyi isolated from diseased Asian seabass (Lates calcarifer). Malaysian J Microbiol. 2012;8(2):104-15.

13. Yingxue $Q$, Jun W, Yongquan S, Wang D, Chen X. Studies on the pathogenic bacterium of ulcer disease in Epinephelus awoara. Acta Oceanologica Sinica. 2018;1:154-9.

14. Shen GM, Shi CY, Fan C, Jia D, Wang SQ, Xie GS, et al. Isolation, identification and pathogenicity of Vibrio harveyi, the causal agent of skin ulcer disease in juvenile hybrid groupers Epinephelus fuscoguttatus $x$ Epinephelus lanceolatus. J Fish Dis. 2017;40:1351-62.

15. Xu X, Liu K, Wang S, Guo W, Xie Z, Zhou Y. Identification of pathogenicity, investigation of virulent gene distribution and development of a virulent strain-specific detection PCR method for Vibrio harveyi isolated from Hainan Province and Guangdong Province. China Aquaculture. 2017:468:226-34.

16. Yano Y, Hamano K, Satomi M, Tsutsui I, Ban M, Aue-Umneoy D. Prevalence and antimicrobial susceptibility of Vibrio species related to food safety isolated from shrimp cultured at inland ponds in Thailand. Food Control. 2014;38(4):30-6.

17. Letchumanan V, Yin WF, Lee LH, Chan KG. Prevalence and antimicrobial susceptibility of Vibrio parahaemolyticus isolated from retail shrimps in Malaysia. Front Microbiol. 2015;6:33.

18. Devi R, Surendran PK, Chakraborty K. Antibiotic resistance and plasmid profiling of Vibrio parahaemolyticus isolated from shrimp farms along the southwest coast of India. World J Microb Bio. 2009;25(11):2005-12.

19. Ji H, Chen Y, Guo Y, Liu X, Wen J, Liu H. Occurrence and characteristics of Vibrio vulnificus in retail marine shrimp in China. Food Control. 2011;22(12): 1935-40.

20. Letchumanan V, Pusparajah P, Tan LTH, Yin WF, Lee LH, Chan KG. Occurrence and antibiotic resistance of Vibrio parahaemolyticus from shellfish in Selangor. Malaysia Front Microbiol. 2015;6:1417.

21. Yano $Y$, Yokoyama M, Satomi M, Oikawa H, Chen SS. Occurrence of Vibrio vulnificus in fish and shellfish available from markets in China. J Food Prot. 2004;67(8):1617-23.

22. Baker-Austin C, Mcarthur JV, Tuckfield RC, Najarro M, Lindell AH, Gooch J, et al. Antibiotic resistance in the shellfish pathogen Vibrio parahaemolyticus isolated from the coastal water and sediment of Georgia and South Carolina, USA. J Food Protect. 2008;71:2552-8.

23. Kang CH, Shin YJ, Kim WR, Kim YG, Song KC, Oh EG, et al. Prevalence and antimicrobial susceptibility of Vibrio parahaemolyticus, isolated from oysters in Korea. Environ Sci Pollut R. 2016;23(1):918-26.

24. Lopatek M, Wieczorek K, Osek J. Prevalence and antimicrobial resistance of Vibrio parahaemolyticus isolated from raw shellfish in Poland. J Food Protect. 2015;78(5):1029-33.

25. Scarano C, Spanu C, Ziino G, Pedonese F, Dalmasso A, Spanu V, et al. Antibiotic resistance of Vibrio species isolated from Sparus aurata reared in Italian mariculture. New Microbiol. 2014;37(3):329-37.

26. Oh EG, Son KT, Yu H, Lee TS, Lee HJ, Shin S, et al. Antimicrobial resistance of Vibrio parahaemolyticus and Vibrio alginolyticus strains isolated from farmed fish in Korea from 2005 through 2007. J Food Protect. 2011;74(3):380-6.

27. He Y, Jin L, Sun F, Hu Q, Chen L. Antibiotic and heavy-metal resistance of Vibrio parahaemolyticus isolated from fresh shrimps in Shanghai fish markets. China Environ Sci Pollut R. 2016;23(15):15033-40.

28. Nakayama T, Ito E, Nomura N, Nomura N, Matsumura M. Comparison of Vibrio harveyi strains isolated from shrimp farms and from culture collection in terms of toxicity and antibiotic resistance. FEMS Microbiol Lett. 2010; 258(2):194-9.

29. Austin $\mathrm{B}$, Zhang $\mathrm{XH}$. Vibrio harveyi: a significant pathogen of marine vertebrates and invertebrates. Lett Appl Microbiol. 2006;43(2):119-24.

30. Natrah FM, Ruwandeepika HA, Pawar S, Karunasagar I, Sorgeloos P, Bossier $P$, et al. Regulation of virulence factors by quorum sensing in Vibrio harveyi. Vet Microbiol. 2011;154(1-2):124-9.

31. De GP, Wright GD. Antibiotic resistance by enzyme inactivation: from mechanisms to solutions. Chembiochem. 2010;11(10):1325-34.

32. Martins A, Spengler G, Molnár J, Amaral L. Bacterial Antibiotic Resistance 2014. p. eLS.
33. Sobecky PA, Hazen TH. Horizontal gene transfer and mobile genetic elements in marine systems. Methods Mol Biol. 2009;532:435-53.

34. Park J, Zhang Y, Bouboltz AM, Zhang XQ, Schuster SC, Ahuja U, et al. Comparative genomics of the classical Bordetella subspecies: the evolution and exchange of virulence-associated diversity amongst closely related pathogens. BMC Genomics. 2012;13(1):545.

35. Le PT, Ramulu HG, Guijarro L, Paganini J, Gouret P, Chabrol O, et al. An automated approach for the identification of horizontal gene transfers from complete genomes reveals the rhizome of Rickettsiales. BMC Evol Biol. 2012; 12(1):243.

36. Jiang W, Marraffini LA. CRISPR-Cas: new tools for genetic manipulations from bacterial immunity systems. Annu Rev Microbiol. 2015;69(1):209-28.

37. Vasu K, Nagaraja V. Diverse functions of restriction-modification systems in addition to cellular defense. Microbiol Mol Biol R Mmbr. 2013;77(1):53-72.

38. Karakulska J, Kaczyn'Ska M, Furowicz AJ, Fijałkowski K. Mobile genetic elements as carriers of antimicrobial resistance genes and transfer of drug resistance in Acinetobacter spp.-selected problems. Advan Agricul Sci. 2010; 5(1):73-81.

39. Hu HW, Wang JT, Li J, Li JJ, Ma YB, Chen D, et al. Field-based evidence for copper contamination induced changes of antibiotic resistance in agricultural soils. Environ Microbiol. 2016;18:3896-909.

40. Sun $M$, Ye M, Wu J, Feng Y, Wan J, Tian D, et al. Positive relationship detected between soil bioaccessible organic pollutants and antibiotic resistance genes at dairy farms in Nanjing. Eastern China Environ Pollut. 2015;206:421-8

41. Jiang K, Xu LW, Su YL, Ma HL, Liu GF, Guo ZX, et al. Isolation and identification of two pathogenetic Vibrio harveyi strains from pearl gentian. Ecol Sci. 2017;36:16-24.

42. Coulthurst SJ. The type VI secretion system-a widespread and versatile cell targeting system. Res Microbiol. 2013;164(6):640-54.

43. Soucy SM, Huang J, Gogarten JP. Horizontal gene transfer: building the web of life. Nat Rev Genet. 2015;16(8):472-82.

44. Majiduddin FK, Materon IC, Palzkill TG. Molecular analysis of beta-lactamase structure and function. Int J Med Microbiol. 2002;292(2):127-37.

45. Confer AW, Ayalew S. The OmpA family of proteins: roles in bacterial pathogenesis and immunity. Vet Microbiol. 2013;163(3-4):207-22.

46. Calamari D, Zuccato E, Castiglioni S, Bagnati R, Fanelli R. Strategic survey of therapeutic drugs in the rivers Po and Lambro in northern Italy. Environ Sci Technol. 2016;37:1241-8

47. Li R, Ye L, Wong MHY, Zheng Z, Chan EWC, Chen S. Evolution and comparative genomics of paqu-like conjugative plasmids in Vibrio species. J Antimicrob Chemoth. 2017;72:2503-6.

48. Klein BU, Siesenop U, Boehm K. Investigations on transferable antibiotic resistance through $\mathrm{R}$-plasmids between obligate and facultative fish pathogenic bacteria. B Eur Assoc Fish Pat. 1996;16:138-42.

49. Lang A, Beatty JT, Rice PA. Guest editorial: Mobile genetic elements and horizontal gene transfer in prokaryotes. Curr Opin Microbiol. 2017;38:V-vii.

50. Liu X, Steele JC, Meng XZ. Usage, residue, and human health risk of antibiotics in Chinese aquaculture: a review. Environ Pollut. 2017;223:161-9.

51. Yu M, Carmichael GR, Zhu T, Cheng Y. Sensitivity of predicted pollutant levels to urbanization in China. Atmos Environ. 2012;60:544-54.

52. Tamang MD, Sunwoo H, Jeon B. Phage-mediated dissemination of virulence factors in pathogenic bacteria facilitated by antibiotic growth promoters in animals: a perspective. Anim Health Res Rev. 2017;18:160-6.

53. Beaber JW, Hochhut B, Waldor MK. SOS response promotes horizontal dissemination of antibiotic resistance genes. Nature. 2004;427(6969):72-4.

54. Mizuno T, Mizushima S. Signal transduction and gene regulation through the phosphorylation of two regulatory components: the molecular basis for the osmotic regulation of the porin genes. Mol Microbiol. 1990;4(7):1077-82.

55. Letchumanan V, Chan KG, Pusparajah P, Saokaew S, Duangjai A, Goh BH, et al. Insights into bacteriophage application in controlling Vibrio species. Front Microbiol. 2016;7:1114

56. Chen WP, Kuo TT. A simple and rapid method for the preparation of gramnegative bacterial genomic DNA. Nucleic Acids Res. 1993;21(9):2260.

57. Delcher AL, Bratke KA, Powers EC, Salzberg SL. Identifying bacterial genes and endosymbiont DNA with Glimmer. Bioinformatics. 2007;23(6):673-9.

58. Lowe TM, Eddy SR. tRNAscan-SE: a program for improved detection of transfer RNA genes in genomic sequence. Nucleic Acids Res. 1997;25(5):955-64.

59. Lagesen K, Hallin P, Rodland EA, Staerfeldt HH, Rognes T, Ussery DW. RNAmmer: consistent and rapid annotation of ribosomal RNA genes. Nucleic Acids Res. 2007;35(9):3100-8. 
60. Griffiths-Jones S. Rfam: an RNA family database. Nucleic Acids Res. 2003; 31(1):439-41.

61. Benson G. Tandem repeats finder: a program to analyze DNA sequences. Nucleic Acids Res. 1999;27(2):573-80.

62. Chen L, Yang J, Yu J, Yao Z, Sun L, Shen Y, et al. VFDB: a reference database for bacterial virulence factors. Nucleic Acids Res. 2005;33:D325-8.

63. Bo L, Mihai P. ARDB-Antibiotic esistance genes database. Nucleic Acids Res. 2009:37:D443-7.

64. Bertelli C, Laird MR, Williams KP, Lau BY, Hoad G, Winsor GL, et al. IslandViewer 4: expanded prediction of genomic islands for larger-scale datasets. Nucleic Acids Res. 2017:45:W30-5.

65. Zhou Y, Liang Y, Lynch KH, Dennis JJ, Wishart DS. PHAST: a fast phage search tool. Nucleic Acids Res. 2011;39:W347-52.

66. Grissa I, Vergnaud G, Pourcel C. CRISPRFinder: a web tool to identify clustered regularly interspaced short palindromic repeats. Nucleic Acids Res. 2007;35:W52-7.

67. Tannistha N, Catherine O, Pratap SA, Justin B, Timothy A, Mitali ST, et al. A genomic survey of positive selection in Burkholderia pseudomallei provides insights into the evolution of accidental virulence. PLoS Pathog. 2010;6(4): e1000845.

68. Qin J, Li R, Raes J, Arumugam M, Burgdorf KS, Manichanh C, et al. A human gut microbial gene catalogue established by metagenomic sequencing. Nature. 2010;464:59-65.

69. Edgar RC. MUSCLE: a multiple sequence alignment method with reduced time and space complexity. Bmc Bioinformatics. 2004;5(1):113-20.

70. Thompson JD, Gibson TJ, Higgins DG. Multiple sequence alignment using clustalw and clustalx. Curr Protoc Bioinformatics. 2002; Chapter 2(Unit 2):Unit 2.3.

71. Srivathsan A, Meier R. On the inappropriate use of Kimura-2-parameter (K2P) divergences in the DNA-barcoding literature. Cladistics. 2012;28(2):190-4.

72. Tamura K, Stecher G, Peterson D, Filipski A, Kumar S. MEGA6: Molecular Evolutionary Genetics Analysis version 6.0. Mol Biol Evol. 2013;30:2725-9.

\section{Publisher's Note}

Springer Nature remains neutral with regard to jurisdictional claims in published maps and institutional affiliations.

Ready to submit your research? Choose BMC and benefit from:

- fast, convenient online submission

- thorough peer review by experienced researchers in your field

- rapid publication on acceptance

- support for research data, including large and complex data types

- gold Open Access which fosters wider collaboration and increased citations

- maximum visibility for your research: over $100 \mathrm{M}$ website views per year

At $\mathrm{BMC}$, research is always in progress.

Learn more biomedcentral.com/submissions 